University of Nebraska - Lincoln

DigitalCommons@University of Nebraska - Lincoln

March 1992

\title{
Hyperspherical Coordinate Description of Single- and Multiphoton Processes in Two-Electron Systems
}

Anthony F. Starace

University of Nebraska-Lincoln, astarace1@unl.edu

Follow this and additional works at: https://digitalcommons.unl.edu/physicsstarace

Part of the Physics Commons

Starace, Anthony F., "Hyperspherical Coordinate Description of Single- and Multiphoton Processes in TwoElectron Systems" (1992). Anthony F. Starace Publications. 125.

https://digitalcommons.unl.edu/physicsstarace/125

This Article is brought to you for free and open access by the Research Papers in Physics and Astronomy at DigitalCommons@University of Nebraska - Lincoln. It has been accepted for inclusion in Anthony F. Starace Publications by an authorized administrator of DigitalCommons@University of Nebraska - Lincoln. 
Chapter to appear in Many-Body Theory of Atomic Structure and Photoionization, edited by Tu-nan Chang (World Scientific Publishing Co., Singapore, 1992)

\title{
HYPERSPHERICAL COORDINATE DESCRIPTION OF SINGLE- AND MULTIPHOTON PROCESSES IN TWO-ELECTRON SYSTEMS
}

\author{
Anthony F. Starace* \\ Joint Institute for Laboratory Astrophysics \\ University of Colorado and National Institute of Standards and Technology \\ Boulder, Colorado 80309-0440 USA
}

\section{Introduction}

While the use of hyperspherical coordinates to describe two-electron systems is quite old [1-8], it was Macek's [9] introduction of an adiabatic approximation in hyperspherical coordinates which made possible a host of theoretical studies elucidating the symmetries of doubly-excited states and the dynamics of processes involving twoelectron atoms and ions. The appeal of the adiabatic hyperspherical approximation is that one can quite literally visualize the relevant physics applicable to complex twoelectron processes by an examination of the shapes of the adiabatic hyperspherical radial potentials. The interpretation of these potentials is straightforward to anyone versed in the analysis of electronic potentials in diatomic molecular problems.

Perhaps because of the powerful insight into two-electron dynamics afforded by the adiabatic hyperspherical representation, most of the theoretical work employing this representation has focused on qualitative interpretation of two-electron processes, the symmetries of excited states of two-electron systems, and the doubly-excited encrgy levels supported by the adiabatic radial potentials. This work, up to about 1982, has been reviewed by Fano [10]. More recently, Lin $[11,12]$ has reviewed the use of the adiabatic hyperspherical approximation for studying the symmetries of doubly-excited states; Starace [13] has provided a critical assessment of the quantitative accuracy of the adiabatic hyperspherical approximation; and Cavagnero [14] has reviewed the application of the hyperspherical coordinate representation to $\mathrm{N}$-electron systems having $N>2$. Beginning in the 1980's, and especially since these recent reviews appeared, there has been increasing use of the adiabatic hyperspherical approximation to calculate cross sections for single and multiphoton processes. Results of these calculations appear to be competitive with those of alternative methods which include electron correlation effects. Furthermore, this quantitative success appears likely to stimulate efforts to improve further upon the adiabatic hyperspherical approximation by treating nonadiabatic effects within more comprehensive theoretical approaches.

The purpose of this chapler is to review the use of the adiabatic hyperspherical approximation for the quantitative calculation of single- and multiphoton cross sections of two-electron systems. In Section 2 we provide a brief general orientation to the hyperspherical coordinate representation, the adiabatic approximation, and a diabatic approximation for treating strongly avoided crossings between adiabatic potential curves. In Section 3 we review applications to photoionization and photodetachment

"JILA Visiting Fellow, on leave from the Department of Physics and Astronomy, the University of Nebraska, Lincoln, NF, 68588-0111. 
processes in $\mathrm{Ile}, \mathrm{Be}, \mathrm{II}^{-}$, and $\mathrm{Ps}^{-}$. In Section 4 we review applications to multiphoton processes in $\mathrm{H}^{-}$. Finally, in Section 5 we assess the adiabatic hyperspherical approximation and discuss future prospects for going beyond this approximation.

\section{The Hyperspherical Representation}

\subsection{Gencral Oricntation}

A two clectron wave function $\psi\left(\vec{r}_{1}, \vec{r}_{2}\right)$ is usually described by the six coordinates $r_{1}, r_{2}, \hat{r}_{1}$, and $\hat{r}_{2}$ of the two clectrons. In hyperspherical coordinates the magnitudes of the individual radial coordinates, $r_{1}$ and $r_{2}$, are replaced by a hyperspherical radius, $R$, and a hyperspherical angle, $\alpha$, where

$$
R \equiv\left(r_{1}^{2}+r_{2}^{2}\right)^{1 / 2}
$$

and

$$
\alpha \equiv \arctan \left(r_{2} / r_{1}\right) \text {. }
$$

The radius $R$ measures the "size" of the two-electron state, while the angle $\alpha$ measures the radial correlation of the two electrons. Note that when $\alpha=\pi / 4, r_{1}=r_{2}$; when $\alpha \approx 0$ or $\approx \pi / 2$, one of the electrons is at a much larger distance from the nucleus than the other.

Before summarizing the features of the Schrödinger equation in these coordinates let us look first at plots of approximate two-electron probabilities $\left|\psi\left(R, \alpha, \hat{r}_{1}, \hat{r}_{2}\right)\right|^{2}$ in these coordinates. Figure 1 shows contour plots $[15,16]$ and Fig. 2 shows relicf maps [17] for the probability distributions of the singly-excited state $1 \mathrm{~s} 2 \mathrm{~s}{ }^{1} \mathrm{~S}$ and the doubly-excited state $2 \mathrm{~s}^{2}{ }^{1} \mathrm{~S}$ of He. (Note that the wave functions are calculated in the approximation that each electron has an orbital angular momentum equal to zero in order to eliminate all dependence on the angular variables $\hat{r}_{1}$ and $\hat{r}_{2}$; since the angular dependence is trivial, these states are symmetric about $\alpha=\pi / 4$, i.e., under interchange of $r_{1}$ and $r_{2}$.) The most obvious distinguishing features of the two probability distributions are that the one for the singly excited state is largest along $\alpha \approx 0$ and $\alpha \approx \pi / 2$ (implying one electron is much further from the nucleus than the other) while the one for the doubly excited state is largest along $\alpha \approx \pi / 4$ (implying both electrons are comparably excited, i.e., $\alpha=\pi / 4$ when $r_{1}=r_{2}$ ). Other important features concern the bchavior of the nodal lines for the two probability distributions. The $1 \mathrm{~s} 2 \mathrm{~s}{ }^{1} \mathrm{~S}$ state has a single nodal line along $R \approx 2$, while the $2 \mathrm{~s}^{2}{ }^{1} \mathrm{~S}$ state has two nodal lincs along $\alpha \approx$ constant: one along $5^{\circ}<\alpha<30^{\circ}$ and the other along $60^{\circ}<\alpha<85^{\circ}$. The fact that the pattern of nodal lines is approximately along the orthonormal grid of constant $R$ and constant $\alpha$ implies a quasi-separability of $R$ and $\alpha$ coordinates.

The nodal line pattern for a particular state serves also to classify the state [17]. The ground statc of $I I c, 1 \mathrm{~s}^{2}{ }^{1} \mathrm{~S}$, has a spherically symmetric probability distribution and is the first member of the singly-excited channel 1 sns ${ }^{1} \mathrm{~S}$, which converges to the $\mathrm{IIe}^{+}(\mathrm{n}=1)$ threshold. The single node in $R$ for the state $1 \mathrm{~s} 2 \mathrm{~s}{ }^{1} \mathrm{~S}$, shown in Figs. 1(a) and 2(a), characterizes it as the second member of the $1 \mathrm{sns}^{1} \mathrm{~S}$ channel. The state $2 \mathrm{~s}^{2}$ ${ }^{1} \mathrm{~S}$, shown in Figs. 1(b) and 2(b), has no radial nodes. It is the first member of the 

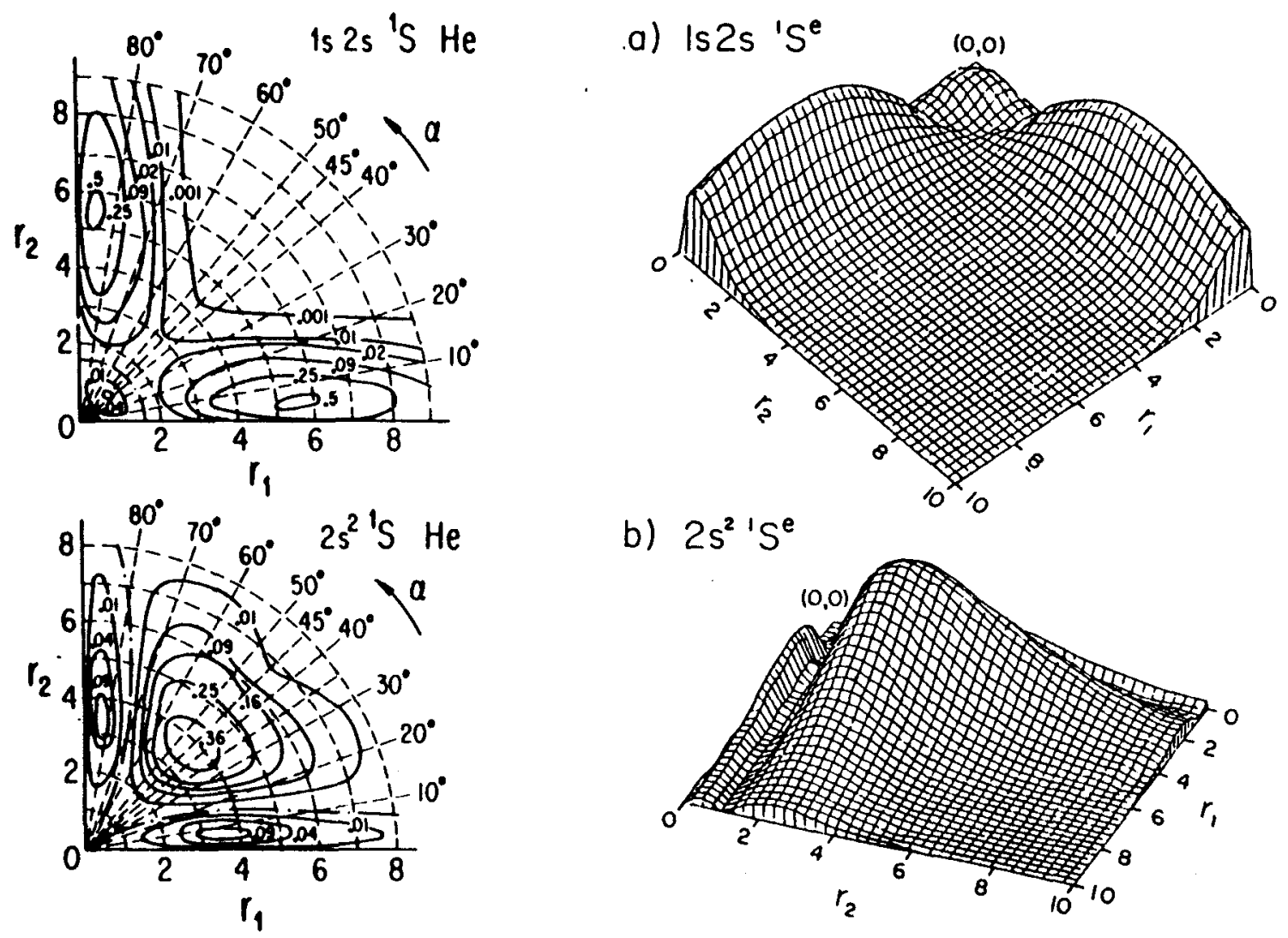

b) $2 s^{2} s^{e}$

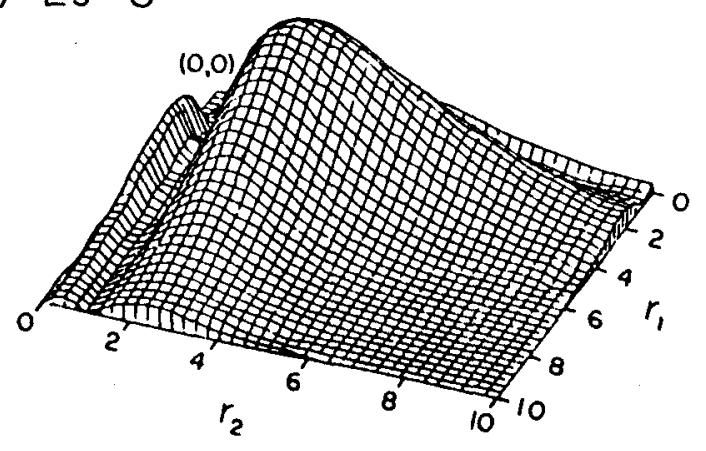

Fig. 1. Contour plot of the approximate probability distribution $\left|\psi\left(\vec{r}_{1}, \vec{r}_{2}\right)\right|^{2}$ for He. (a) $1 \mathrm{~s} 2 \mathrm{~s}^{1} \mathrm{~S}$ (b) $2 \mathrm{~s}^{2}{ }^{1} \mathrm{~S}$. Solid Lines: lines of constant probability. Dot-Dash Lines:

Fig. 2. Relief map of the approximate probability distribution $\left|\psi\left(\vec{r}_{1}, \vec{r}_{2}\right)\right|^{2}$ for He. (a) $1 \mathrm{~s} 2 \mathrm{~s}^{1} \mathrm{~S}$ (b) $2 \mathrm{~s}^{2}{ }^{1} \mathrm{~S}$ (From Ref. [17].) nodal lines. (From Ref. [16].)

Rydberg series 2 sns ${ }^{1} \mathrm{~S}$ converging to the $\mathrm{He}^{+}(\mathrm{n}=2)$ threshold. The two nodes approximately along constant $\alpha$, symmetrical about $\alpha=\pi / 4$, characterize $2 \mathrm{~s}^{2}{ }^{1} \mathrm{~S}$ as a member of this second Rydberg channel. Thus nodes in $R$ characterize the excitation of a state within a channel while nodes in $\alpha$ characterize the various channels [17].

\subsection{Two-Electron Schrödinger Equation}

In hyperspherical coordinates the nonrelativistic two-electron Schrödinger equation becomes

$$
\begin{aligned}
\left(\frac{d^{2}}{d R^{2}}\right. & \left.-\frac{1}{R^{2}}\left(-\frac{d^{2}}{d \alpha^{2}}-\frac{1}{4}+\frac{\ell_{1}^{2}}{\sin ^{2} \alpha}+\frac{\ell_{2}^{2}}{\cos ^{2} \alpha}\right)-\frac{C\left(\alpha, \theta_{12}\right)}{R}+2 E\right) \\
& \times\left(R^{5 / 2} \sin \alpha \cos \alpha \psi\right)=0
\end{aligned}
$$


where the potential $-C\left(\alpha, \theta_{12}\right)$ is proportional to the sum of the nuclear and electrostatic potentials,

$$
\begin{aligned}
-C\left(\alpha, \theta_{12}\right) & =R\left(-\frac{2 Z}{r_{1}}-\frac{2 Z}{r_{2}}+\frac{2}{\left|\vec{r}_{2}-\vec{r}_{1}\right|}\right) \\
& =-\frac{2 Z}{\cos \alpha}-\frac{2 Z}{\sin \alpha}+\frac{1}{\left(1-\sin 2 \alpha \cos \theta_{12}\right)^{1 / 2}},
\end{aligned}
$$

$\vec{\ell}_{1}$ and $\vec{\ell}_{2}$ are the usual orbital angular momentum operators for the individual electrons, $\theta_{12} \equiv \cos ^{-1} \hat{r}_{1} \cdot \hat{r}_{2}$, and $Z$ is the nuclear charge.

In the hyperspherical coordinate method of Macek [9], the two-electron wave function $\psi_{\nu}\left(\vec{r}_{1}, \vec{r}_{2}\right)$ is expanded in terms of a complete set of adiabatic eigenfunctions $\phi_{\mu}\left(R ; \alpha, \hat{r}_{1}, \hat{r}_{2}\right)$, which depend parametrically on the hyperspherical radius $R$ and are functions of the five angular variables $\alpha, \hat{r}_{1}$, and $\hat{r}_{2}$. The form of $\psi_{\nu}$ is thus:

$$
\psi_{\nu}\left(R, \alpha, \hat{r}_{1}, \hat{r}_{2}\right)=\left(R^{5 / 2} \sin \alpha \cos \alpha\right)^{-1} \sum_{\mu} F_{\mu \nu}(R) \phi_{\mu}\left(R ; \alpha ; \hat{r}_{1}, \hat{r}_{2}\right) .
$$

The angular function $\phi_{\mu}$ is defined to satisfy the following differential equation in atomic units $(\hbar=c=m=1)$ :

$$
\left(-\frac{d^{2}}{d \alpha^{2}}+\frac{\ell_{1}^{2}}{\cos ^{2} \alpha}+\frac{\ell_{2}^{2}}{\sin ^{2} \alpha}-R C\left(\alpha, \theta_{12}\right)\right) \phi_{\mu}=-U_{\mu}(R) \phi_{\mu} .
$$

Ilere $-C\left(\alpha, 0_{12}\right)$ is defined in Eq. (4) and $U_{\mu}(R)$ is an eigenvalue which is parametrically dependent on $R$. Upon substituting Eq. (5) in the two-electron Schrödinger cquation and using Eq. (6), one obtains the following set of coupled differential equations for the radial functions $F_{\mu \nu}(R)$ :

$$
\begin{aligned}
\left(\frac{d^{2}}{d R^{2}}\right. & \left.+\frac{U_{\mu}(R)+\frac{1}{4}}{R^{2}}+\left(\phi_{\mu}, \frac{\partial^{2} \phi_{\mu}}{\partial R^{2}}\right)+2 E\right) F_{\mu \nu}(R) \\
& +\sum_{\mu^{\prime} \neq \mu}\left(\left(\phi_{\mu}, \frac{\partial^{2} \phi_{\mu^{\prime}}}{\partial R^{2}}\right)+2\left(\phi_{\mu}, \frac{\partial \phi_{\mu^{\prime}}}{\partial R}\right) \frac{\partial}{\partial R}\right) F_{\mu^{\prime} \nu}(R)=0 .
\end{aligned}
$$

In Eq. (7) the coupling matrix elements $\left(\phi_{\mu}, \partial^{n} \phi_{\mu}, / \partial R^{n}\right), n=1,2$, involve integration over the five angular variables only and are thus parametrically dependent on $R$.

\subsection{The Adiabatic Approximation}

Each of the potentials $U_{\mu}(R)$ and its corresponding angular eigenfunction $\phi_{\mu}$ define a hyperspherical channel $\mu$. These channels are coupled through the radial derivative matrix elements in Eq. (7). In the adiabatic hyperspherical approximation [9], one ignores the coupling terms in the second set of braces in Eq. (7). Then the wave 
function in Eq. (5) may be represented by a single term with $\mu=\nu$ in the summation on the right side, i.e.,

$$
\psi_{\mu E}^{a d}=\left(R^{5 / 2} \sin \alpha \cos \alpha\right)^{-1} F_{\mu \mu E}(R) \phi_{\mu}\left(R ; \alpha, \hat{r}_{1}, \hat{r}_{2}\right)
$$

For simplicity one usually sets $\mu=\nu$ and drops the double subscripts on $\mathrm{F}$ when referring to the adiabatic approximation solutions. One sees from Eq. (8) that the adiabatic approximation amounts to assuming that motion in $\mathrm{R}$ and motion in $\alpha$ are approximately independent of each other. This quasi-separability was inferred from Figs. 1 and 2, which show electron density plots obtained from quasi-separable approximation wave functions. This behavior may be confirmed by examining correlated two-electron wave functions and observing that the nodal lines of such wave functions also lie approximately along constant $R$ and along constant $\alpha$ [18].

It should be emphasized that although only single radial and angular functions are used to represent the two-electron wave function in Eq. (8), much electron correlation is implicitly included. This is illustrated in Fig. 3, which shows the $\mathrm{s}^{2}\left({ }^{1} \mathrm{~S}\right), \mathrm{p}^{2}\left({ }^{1} \mathrm{~S}\right)$, $\mathrm{d}^{2}\left({ }^{1} \mathrm{~S}\right)$, and $\mathrm{f}^{2}\left({ }^{1} \mathrm{~S}\right)$ components of the numerically calculated $\mathrm{H}^{-}\left({ }^{1} \mathrm{~S}\right)$ ground state
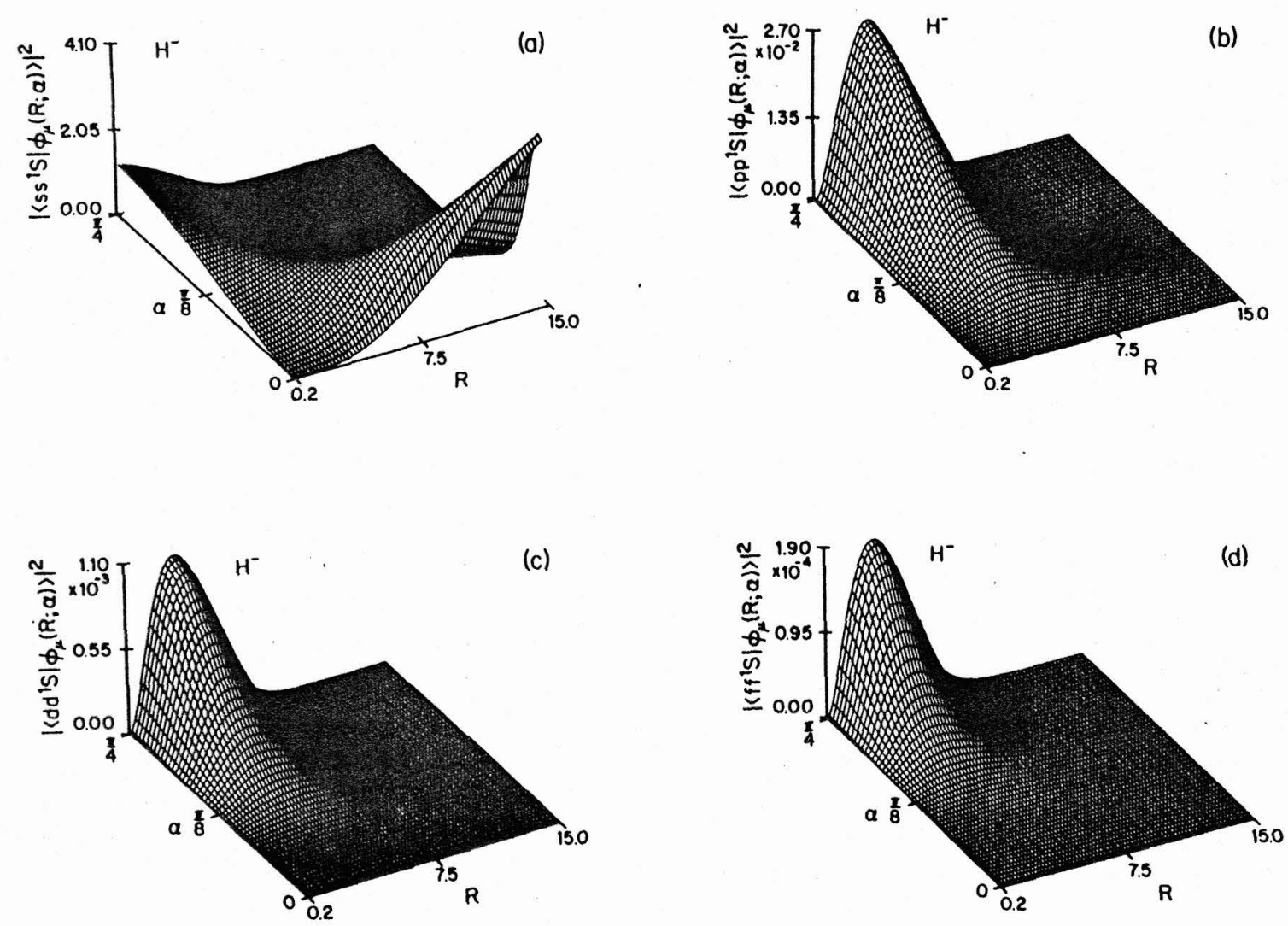

Fig. 3. Probability (per unit length in $\alpha$ ) for the angular momentum state $\ell \ell^{1} S$ in the singlet ground state of $\mathrm{H}^{-}$for (a) $\ell=0$; (b) $\ell=1$; (c) $\ell=2$; (d) $\ell=3$. Note multiplication by factors of $10^{2}, 10^{3}$, and $10^{4}$ in Figs. (b), (c), and (d), respectively. (From Ref. [19].) 
angular function $\phi_{\mu}$ [19]. One sees clearly that these higher angular momentum components are significant at small $R$, near $\alpha \approx \pi / 4$ (i.e., $r_{1} \approx r_{2}$ ). As $R$ increases, however, only the ss $\left({ }^{1} \mathrm{~S}\right)$ component contributes significantly, in accordance with the independent, electron model.

Notice also in Eq. (8) how all members of the channel $\mu$ have the same angular function $\phi_{\mu}$ at any given $R$. Each state of excitation energy $E$ within the channel $\mu$ is described by the radial function $F_{\mu E}$, which is calculated in the channel potential $U_{\mu}(R)$ using $\mathrm{Fq}$. (7) and ignoring the off-diagonal coupling terms. Because each momber of a Rydberg scries of doubly excited states has the same angular function $\phi_{\mu}$ and has a radial function $F_{\mu E}(R)$ that is calculated in the same potential $U_{\mu}(R)$, the physical properties of states belonging to a particular channel $\mu$ are often immediately apparent upon examination of $U_{\mu}(R)$ and $\phi_{\mu}$, as we illustrate next by the first application to a photoionization proccss.

\subsection{Interpretation of the IIe Photoabsorption Spectrum Below the $n=2$ Threshold}

The first major success [9] of the adiabatic approximation in hyperspherical coordinates was the classification and interpretation of the photoabsorption spectrum of He in the region of the doubly excited Rydberg states converging to the $n=2$ threshold. In the usual classification scheme there should be three Rydberg series of such levels of comparable intensity: $2 \mathrm{snp}^{1} \mathrm{P}, 2 \mathrm{pnd}{ }^{1} \mathrm{P}$, and $2 \mathrm{pns}{ }^{1} \mathrm{P}$. The experimental spectrum of Madden and Codling [20] showed only one strong Rydberg series and one very weak Rydberg series. The third possible series was not observed. Cooper, Fano and Prats [21] interpreted the relative intensitics of the two observed series in terms of the so-called " + " and " " series, $(2 \mathrm{snp} \pm 2 \mathrm{pns})^{1} \mathrm{P}$. The " + " series members are more intense than those of the "-" series because the corresponding wave functions of the " + " members have a much larger amplitude near the origin, allowing therefore a much larger overlap with the ground state. This scheme, however, does not explain the weakness of the 2 pnd $^{1} \mathrm{P}$ channel. Figure 4, however, shows Macek's hyperspherical potentials $U_{\mu}(R)$ for the three ${ }^{1} \mathrm{p}^{\circ}$ channels $\mu$ converging to the $n=2$ state of $\mathrm{He}^{+}$. One sees immediately that the three channels have vastly different centrifugal barriers near the origin, explaining the large intensity differences of the three allowed channels. Furthermore, the first two hyperspherical channels have the "+" and "-" characteristics predicted by Cooper et al. [21].

\subsection{Adiabalic vs. Diabalic Potentials}

Thus, the experimental observation of only a single intense Rydberg series converging to the $n=2$ threshold in the photoabsorption spectrum of He can be understood easily in terms of the overlap of the initial and the final wave functions. The potential labelled " 1 " is the so-called "+" channel, whose states overlap the ground state much more effectively than do states in either the " 2 " or "-" potential or the " 3 " or " $\mathrm{d}$ " potential. Nole however, that the " 1 " and " 2 " potentials cross at $R \approx 7.64$. 


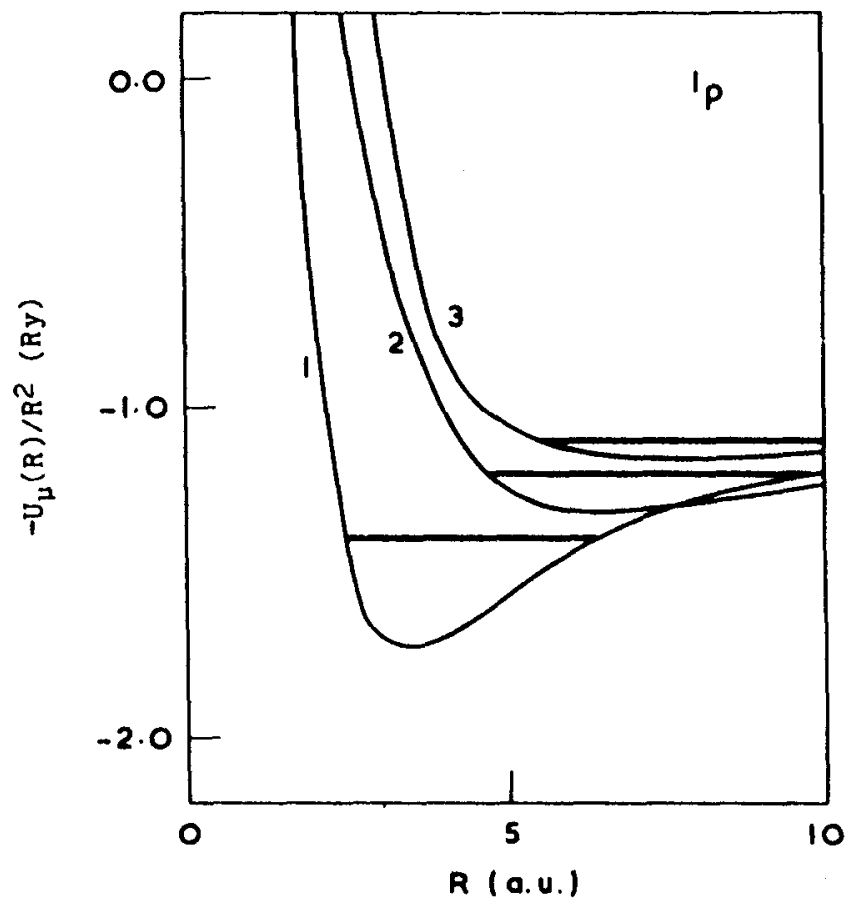

Fig. 4. Hyperspherical potential curves $-U_{\mu} / R^{2}$ vs. $R$ for the three He doubly excited ${ }^{1} \mathrm{P}^{o}$ channels converging to the $n=2$ state of $\mathrm{He}^{+}$. (From Ref. [9].)

Figure 5, which examines this region in greater detail, shows that the adiabatic approximation potentials actually do not cross, but have instead a sharply avoided crossing. Because of this avoided crossing over a small region in $R$, the angle functions $\phi_{\mu}$ have large derivatives with respect to $R$. In fact, the coupling matrix elements are so large that the " + " and "-" potentials exchange their character for $R>7.64$. Figure 6 shows this exchange by plotting the $R$-dependence of the overlap integral of $\phi_{-}(R=6.5)$ with $\phi_{-}(R)$ and with $\phi_{+}(R)$. Whereas for $R<7.64,<\phi_{-}(6.5) \mid \phi_{-}(R)>$ is close to unity, as expected, and $\left\langle\phi_{-}(6.5) \mid \phi_{+}(R)\right\rangle$ is close to zero, one finds that for $R>7.64,<\phi_{-}(6.5)\left|\phi_{+}(R)\right\rangle$ is close to unity and $\left\langle\phi_{-}(6.5) \mid \phi_{-}(R)\right\rangle$ is close to zero. What is happening is that electronic excitations populated in the " + " channel at small $R$ proceed outward at larger $R$ and "hop" from the adiabatic " + " channel to the "_" channel near $R \approx 7.64$. For this reason one usually employs the diabatic approximation shown in Fig. 5 in such cases of sharply avoided crossings. That is, one connects the " $t$ " potential and channel function below $R \approx 7.64$ to the "-" potential and channel function above $R \approx 7.64$ and vice versa. One then ignores the residual coupling between the new " + " and "-" diabatic potentials. In general, "adiabatic" hyperspherical calculations make use of the diabatic potential curves in cases involving such sharply avoided crossings. 


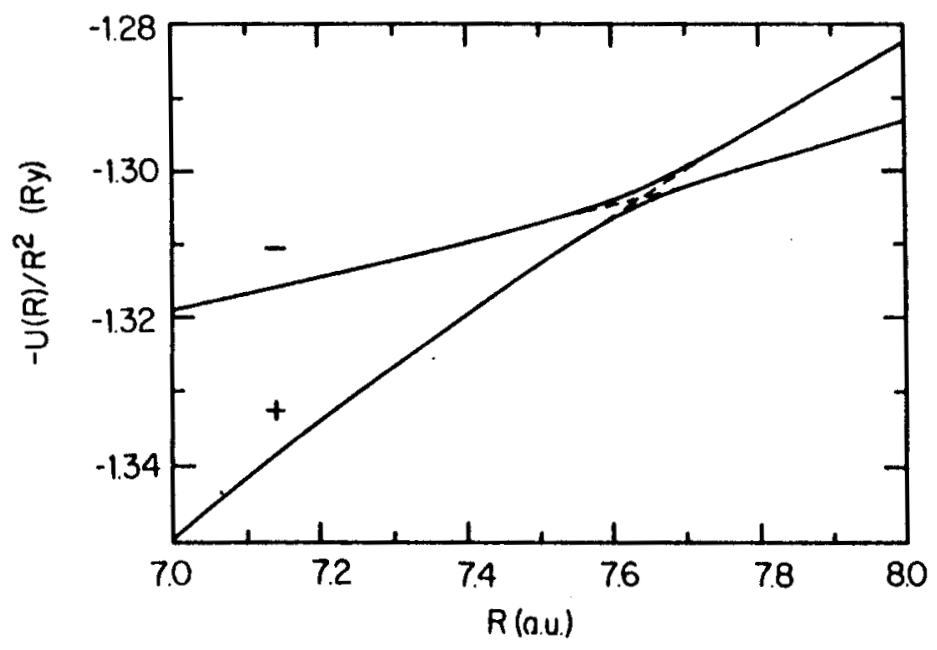

Fig. 5. The $\mathrm{He}^{+}(n=2)-e^{-1}$ po " $+" /$ "-" avoided crossing: a special case of transition from one $U_{\mu}(R)$ to another. Solid lines show the adiabatic hyperspherical potentials, which have an avoided crossing near $R \approx 7.64$. The dashed lines show the diabatic potentials, which cross near $R \approx 7.64$.

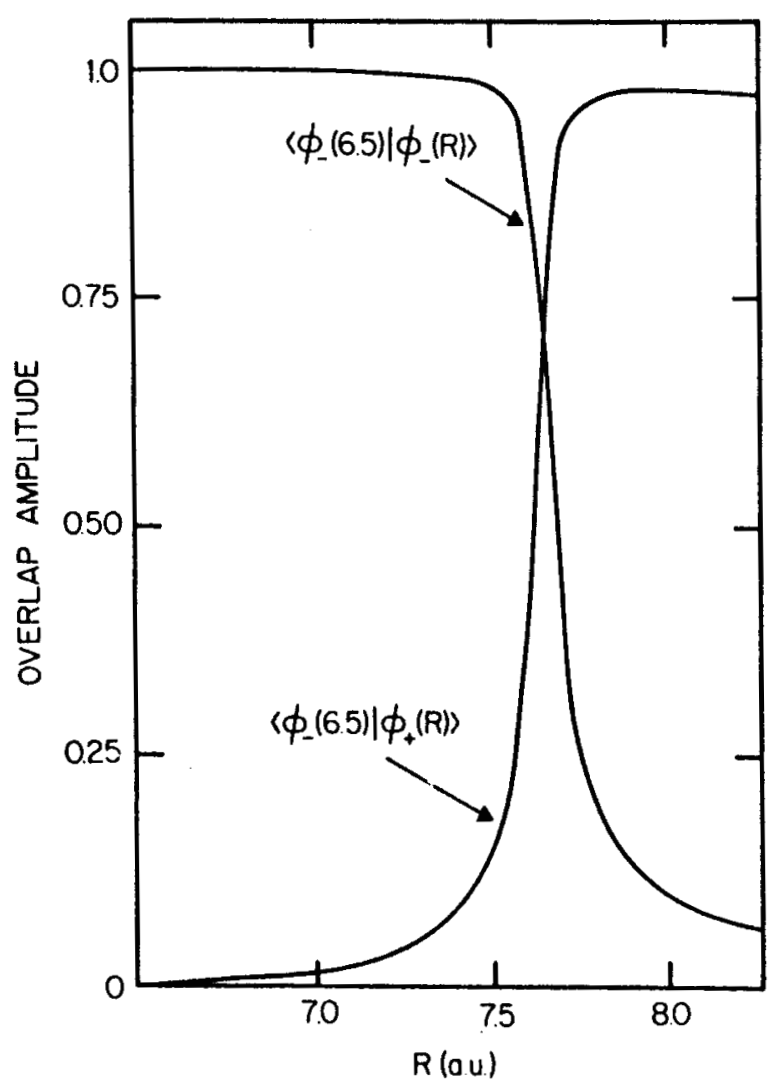

Fig. 6. Adiabatic approximation channel function behavior at the "+"/"-" avoided crossing near $R=7.64$ as exhibited by the $R$-dependence of the two overlap integrals $\left\langle\phi_{-}(R=6.5\right.$ a.u. $\left.) \mid \phi_{-}(R)\right\rangle$ and $\left\langle\phi_{-}(R=6.5\right.$ a.u. $\left.) \mid \phi_{+}(R)\right\rangle$. Integrations are over $\alpha, \hat{r}_{1}$, and $\hat{r}_{2}$. 


\section{Description of Photoionization Processes}

\subsection{Electric Dipole Transition Matrix Elements}

Electric dipole transition matrix elements between adiabatic hyperspherical wave functions are evaluated using standard tensor algebra techniques and the following expansion of the initial and final state channel functions $\phi_{\mu}$ (cf. Eq. 6) in terms of coupled spherical harmonics:

$$
\phi_{1} \equiv \sum_{l_{1} l_{2}} A_{l_{1} l_{2} L M}^{\mu}(R ; \alpha) \mathcal{Y}_{l_{1} l_{2} L M}\left(\hat{r}_{1}, \hat{r_{2}}\right)
$$

where

$$
\mathcal{Y}_{l_{1} l_{2} L M}\left(\hat{r}_{1}, \hat{r}_{2}\right)=\sum_{m_{1} m_{2}} Y_{l_{1} m_{1}}\left(\hat{r}_{1}\right) Y_{l_{2} m_{2}}\left(\hat{r}_{2}\right)\left\langle l_{1} m_{1} l_{2} m_{2} \mid L M\right\rangle .
$$

In Eq. (9) antisymmetry of the wave function is ensured by boundary conditions [9] on the cocfficients $\Lambda_{l_{1} l_{2}}^{\mu}$. Substituting Eqs. (9) and (10) in Eq. (6) gives the following differential equation lor the expansion coefficients:

$$
\begin{aligned}
{\left[\frac{d^{2}}{d \alpha^{2}}-\frac{l_{1}\left(l_{1}+1\right)}{\cos ^{2} \alpha}\right.} & \left.-\frac{l_{2}\left(l_{2}+1\right)}{\sin ^{2} \alpha}-U_{\mu}(R)\right] A_{l_{1} l_{2} L M}^{\mu}(R ; \alpha) \\
& =-R \sum_{l_{1}^{\prime} l_{2}^{\prime}} C_{l_{1} l_{2}, l_{1} l_{2}^{\prime}} A_{l_{1}^{\prime} l_{2}^{\prime} L M}^{\mu}(R ; \alpha),
\end{aligned}
$$

where

$$
C_{l_{1} l_{2}, l_{1}^{\prime} l_{2}^{\prime}} \equiv \int_{0}^{\pi / 2} d \alpha \int d \hat{r}_{1} d \hat{r}_{2} \mathcal{Y}_{l_{1} l_{2} L M}^{*}\left(\hat{r}_{1}, \hat{r}_{2}\right) C\left(\alpha, 0_{12}\right) \mathcal{Y}_{l_{1}^{\prime} l_{2}^{\prime} L M}\left(\hat{r}_{1}, \hat{r}_{2}\right),
$$

and where $C\left(\alpha, 0_{12}\right)$ is defined in Eq. (4).

The dipole matrix element for incident light linearly polarized along the $z$ axis in the length $(L)$ form is defined by

$$
\begin{aligned}
D_{L} & =\left\langle\Psi_{E}\left|\hat{\epsilon}_{z} \cdot \sum_{i=1}^{2} \vec{r}_{i}\right| \Psi_{0}\right\rangle \\
& =\left\langle\Psi_{E}\left|R\left(\cos \alpha \cos \theta_{1}+\sin \alpha \cos \theta_{2}\right)\right| \Psi_{0}\right\rangle \\
& =\sum_{\mu^{\prime} \mu} \int_{0}^{\infty} d R F_{\mu^{\prime} E}(R) R F_{\mu}(R) I_{\mu^{\prime} \mu}^{L}(R) .
\end{aligned}
$$

Ilerc $I_{\mu^{\prime} \mu}^{L}(R)$ denotes the angular integral, whose general expression is presented by Park et al. [22]. We present here the result for the simpler but nevertheless important special case of ${ }^{1} \mathrm{~S} \rightarrow{ }^{1} \mathrm{P}$ transitions:

$$
\begin{aligned}
l_{\mu^{\prime} \mu}^{\prime}(R) & =\sum_{l_{1} l_{2}} 3^{-1 / 2}\left[l_{1}\right]^{-1 / 2}\left(l_{2}\left\|C^{[1]}\right\| l_{1}\right) \\
& \times\left[\int_{0}^{\pi / 2} d \alpha A_{l_{2} l_{1}}^{\mu^{\prime}} \cos \alpha A_{l_{1} l_{1}}^{\mu}+\int_{0}^{\pi / 2} d \alpha A_{l_{1} l_{2}}^{\mu^{\prime}} \sin \alpha A_{l_{1} l_{1}}^{\mu}\right]
\end{aligned}
$$


where

$$
\left(l_{2}\left\|C^{(1]}\right\| l_{1}\right) \equiv(-1)^{l_{2}}\left[l_{1}\right]^{1 / 2}\left[l_{2}\right]^{1 / 2}\left(\begin{array}{ccc}
l_{2} & 1 & l_{1} \\
0 & 0 & 0
\end{array}\right) .
$$

Transition matrix elements for other cases of incident light polarization may be obtained from Eq. (13) by application of the Wigner-Eckart theorem. Park et al. [22] also present adiabatic hyperspherical expressions for the velocity and acceleration forms of the electric dipole matrix elements.

\subsection{Photoionization of IIelium}

The first application of the adiabatic hyperspherical approximation to the calculation of photoionization cross sections was made by Miller and Starace [23], who treated the process:

$$
H e\left({ }^{1} S\right)+\gamma \rightarrow H e^{+} 1 s\left({ }^{2} S\right)+e^{-}\left({ }^{1} P\right) .
$$

Their initial and final wave functions for this process both have the form of Eq. (8). For the initial state, $\mu$ corresponds to the lowest ' $S$ potential $U_{\mu}(R)$, and for the final state, $\mu$ corresponds to the lowest ${ }^{1} \mathrm{P}$ potential $U_{\mu}(R)$. The photoionization cross section obtained using the adiabatic approximation wave functions is shown in Fig. 7. Figure 7 also shows the revised experimental results of Samson [24], which have

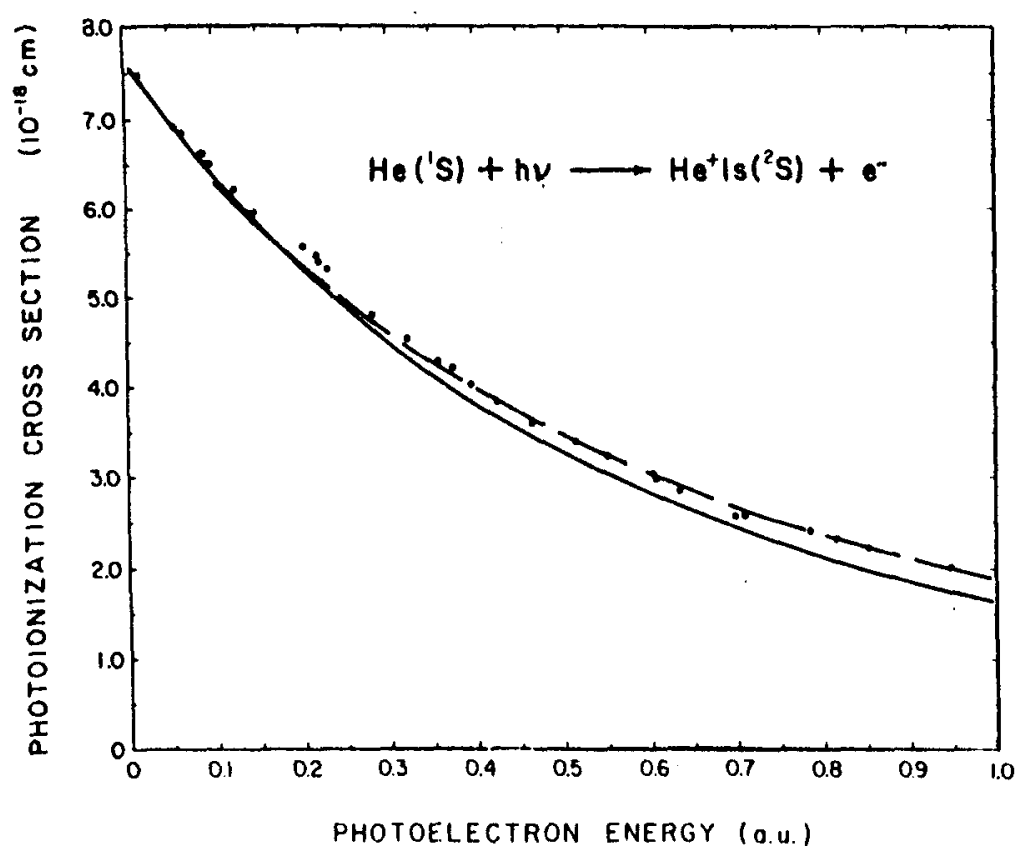

Fig. 7. Photoionization cross section for He. Full Curve: adiabatic approximation (single channel) hyperspherical calculation of Miller and Starace (Ref. [23]); Dots: Experimental results of Samson (Ref. [24]); Dashed Curve: $18-2 \bar{s}-2 \bar{p}$ (four channel) close-coupling calculation of Jacobs (Ref. [25]). (From Ref. [23].) 
crror bars of $\pm 3 \%$. The hypersphcrical results lie within these error limits near threshold (for kinetic energies $0.0 \leq \epsilon \leq 0.4$ a.u.) and in fact agree with experiment to within $1 \%$ at threshold. The hyperspherical results, however, are systematically lower than experiment above $\epsilon=0.4$ a.u. Of the many other theoretical calculations, we show one with very good overall agreement with experiment: the four-channel $(1 \mathrm{~s}-2 \bar{s}-2 \bar{p})$ close-coupling calculation of Jacobs [25]. In comparison with the closecoupling results, the single-channel hyperspherical results are in better agreement with expcriment below $\epsilon=0.2$ a.u. and are systematically lower above $\epsilon=0.2$ a.u. Note that the length results of a more recent six-state $\mathrm{R}$-matrix calculation by Berrington et al. [26] are in excellent agreement with the experimental results of West and Marr [27], including those near threshold, thereby indicating the sophistication required to properly describe the threshold region by methods employing independent electron representations.

\subsection{Photoionization of Bc}

Greene [28] has calculated the photoionization cross section of Be including coupling between the lowest two hyperspherical channels: $\mu=1$, corresponding to leaving the ion in its ground $2 \mathrm{~s}$ state, and $\mu=2$, corresponding to leaving the ion in its excited $2 \mathrm{p}$ state. (The inncr $1 \mathrm{~s}^{2}$ core was represented by a central potential so that only the correlation of the outer two elcctrons was treated.) Greene's procedure is to calculate the two adiabatic potentials $U_{\mu}(R)$ and angle functions $\phi_{\mu}$ using the angular Eq. (6). The radial Eq. (7) is then solved including the first and second derivative coupling matrix elements connecting the channels $\mu=1$ and $\mu=2$. As shown in Fig. 8 , his results are in reasonable agreement with the close-coupling calculation of Dubau and Wells [29] and show a very large intensity for excitation of the ion to the $2 p$ level.

The most interesting aspect of Greene's calculation [28] is the similarity his hyperspherical wave functions show to those in He, thereby indicating a similar bchavior for lle, Bc, and all the alkaline earths. It is instructive first to compare the hyperspherical potentials $U_{\mu}(R)$ for the He ${ }^{1} \mathrm{P}$ levels converging to the $\mu=2$ threshold, shown in Fig. 4, to the corresponding potential curves in Be, shown in Fig. 9. One sees inmedialely from Fig. 4 why only one of the $\operatorname{He}^{+}(n=2)$ excitation channels, $\mu=1$, is strongly populated: it has a much less repulsive potential barrier than either the $\mu=2$ or $\mu=3$ channels. Furthermore, the channel function $\phi_{\mu}$ for the " $t$ " channel $(\mu=1)$ is symmetric in $\alpha$, having an antinode on the well-known [16] potential ridge of Eq. (4) (along $\alpha=\pi / 4)$, while the "-" channel $(\mu=2)$ is antisymmetric in $\alpha$, having a node on the potential ridge. The symmetry about $\alpha=\pi / 4$ for the He wave functions holds for all $R$ values due to the degeneracy in energy of these channels. Reiterating the discussion above, recall that although the " + " and " - " channels are shown to cross in Fig. 4, this crossing is actually avoided; in any case the channel functions $\phi_{\mu}$ do not adjust to the crossing but proceed diabatically through it. For this reason the iniddle curve in Fig. 4 for $R>7.64$ a.u. has " + " character while the lowest curve for $R>7.64$ a.u. has "-" character. 

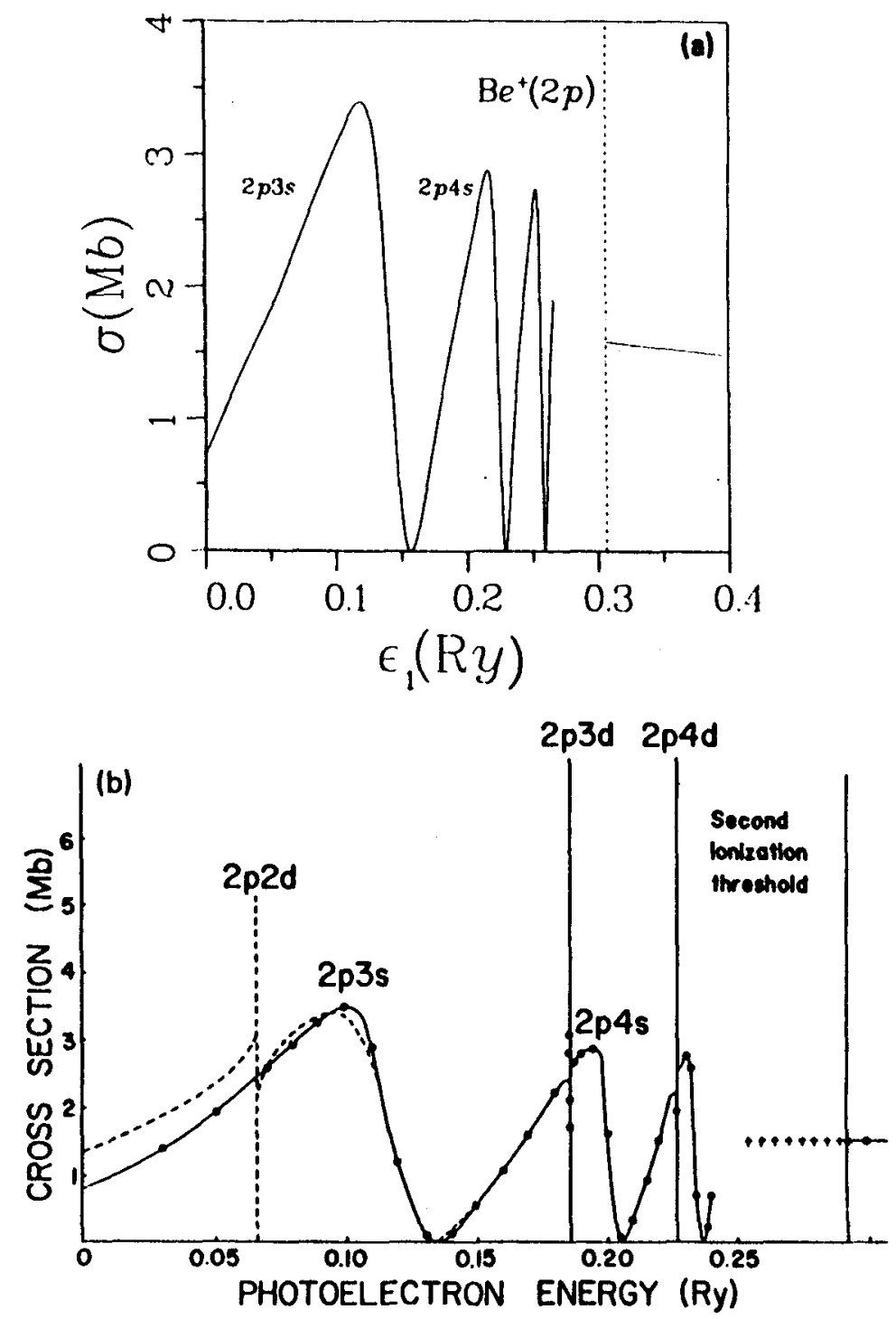

Fig. 8. Photoionization cross section for Be plotted vs. photoelectron energy $\epsilon(R y)$. (a) Coupled channel hyperspherical calculation of Greene [28]. (b) Close-coupling calculation of Dubau and Wells [29].

Consider now the Be potentials in Fig. 9. Two differences from He are immediately apparent. First, the potential curves are nondegenerate for $R \rightarrow \infty$. Second, there is an avoided crossing between the first and second potential curves for $4<R<6$. Otherwise, however, one expects most of the absorption strength, as in He, to go into the channel with the lowest potential curve. In his calculations Greene expanded the channel functions $\phi_{\mu}$ as in Eq. (9) [although he used the notation $g_{\mu}^{\ell_{1} \ell_{2}}(R, \alpha)$ instead of $\left.A_{\ell_{1} \ell_{2} L M}^{\mu}(R, \alpha)\right]$. The most important functions $g_{\mu}^{\ell_{1} \ell_{2}}$ (those with $\ell_{1} \ell_{2}=$ "sp") are shown in Fig. 10 for the potential curves $\mu=1$ and $\mu=2$ for various $R$ values. For $R=2$ one sees that the $\mu=1$ function is approximately symmetric about $\alpha=\pi / 4$ 


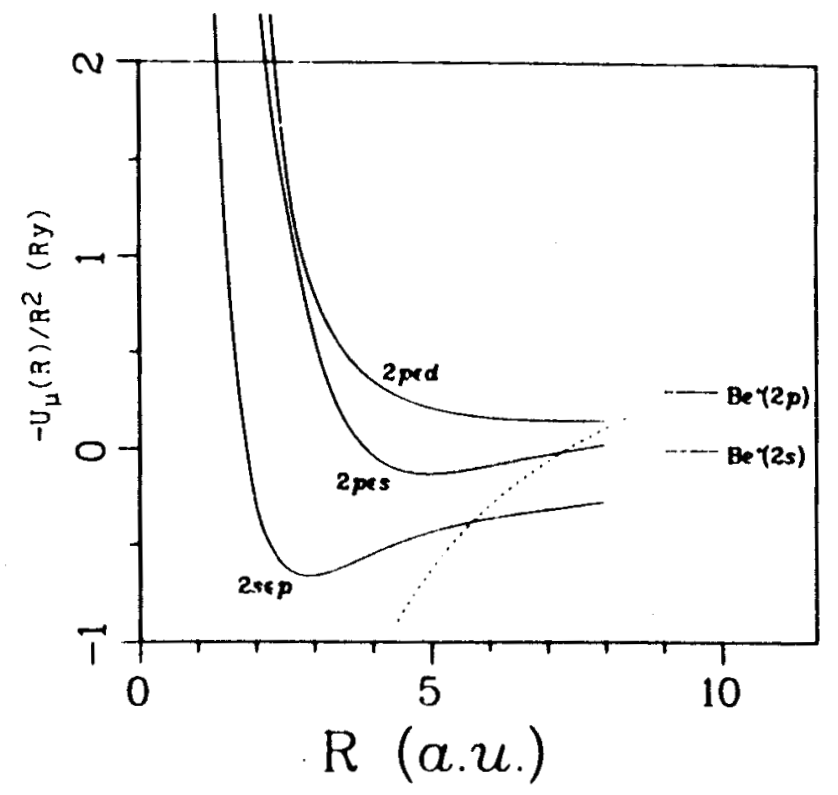

Fig. 9. Hyperspherical potential curves $-U_{\mu}(R) / R^{2}$ vs. $R$ for the three Be ${ }^{1} \mathrm{P}$ channels converging to the $n=2$ state of Be. (From Ref. [28].)

while the $\mu=2$ function is approximately antisymmetric, just as for the " + " and "-" channels in He. As $R$ increases, however, these adiabatic channel functions drop into one or the other of the potential valleys of Eq. (4), i.e., the $\mu=1$ amplitude becomes concentrated near $\alpha=0$ while the $\mu=2$ amplitude becomes concentrated near $\alpha=\pi / 2$. Thus, as $R$ increases the nondegeneracy of the thresholds in $\mathrm{Be}$ causes a breakdown of the " + " and "-" symmetry about $\alpha=\pi / 4$ observed at small $R$ values. Furthermore, this transition is seen to occur for $R$ values $4 \leq R \leq 6$.
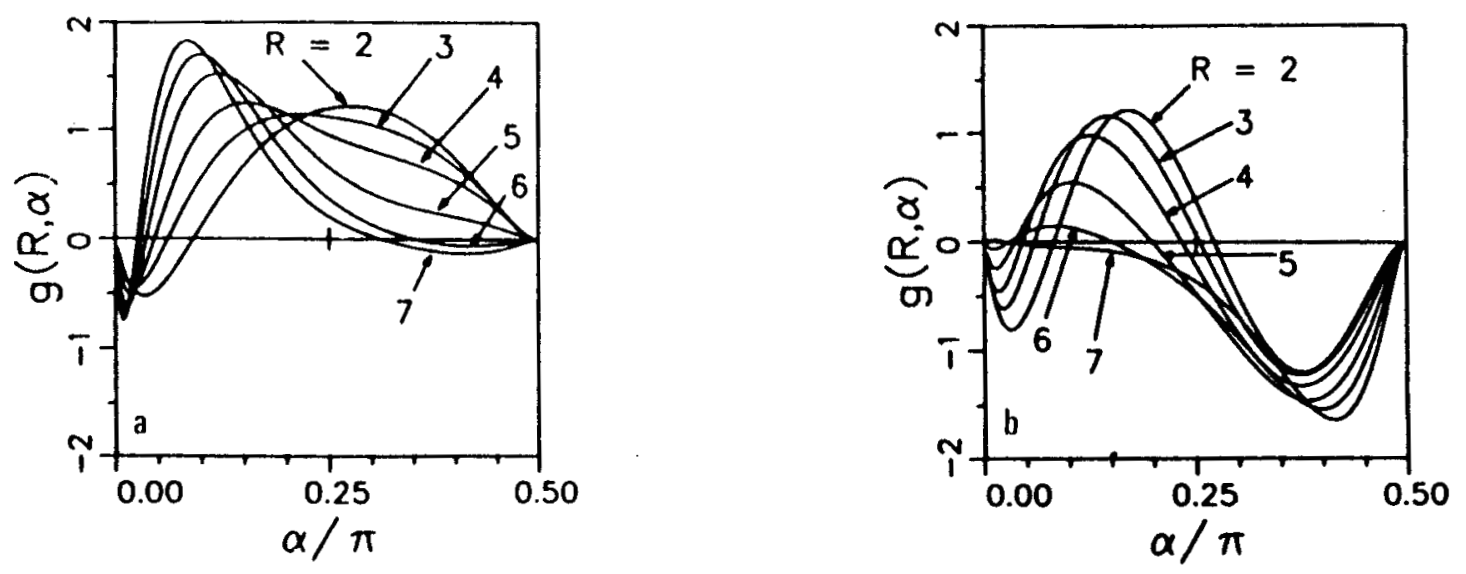

Fig. 10. Adiabatic "sp" channel wave functions associated with the lowest two Be potential curves: (a) $2 \mathrm{sep}, \mu=1$; (b) $2 \mathrm{pes}, \mu=2$. (From Ref. [28].) 
What is remarkable about Greenc's treatment of the coupled radial equations (7) is the finding that the solution which at small $R$ starts out as the adiabatic wave function $F_{\mu=1}(R) \phi_{\mu=1}(R ; \Omega)$ in the $\mu=1$ channel becomes at $R>6$ a nearly equal superposition of the adiabatic wave functions for $\mu=1$ and $\mu=2$ in such a way that the " + " symmetry is preserved through the avoided potential crossing region. In other words, just as in He, the " + " solution proceeds diabatically through the avoided potential crossing. This also explains the large excitation cross section observed in Be since, unlike the case in IIc, the state having " + " character becomes at $R>6$ a nearly cqual supcrposition of the $\mu=1$ and $\mu=2$ channel functions. Furthermore, it is expected that this diabatic behavior of the hyperspherical " + " solution will be a common feature of all alkaline earth and other similar two electron systems [10, 28].

Indecd, R-matrix calculations [30] have found the eigenchannel functions for $\mathrm{Mg}$ 'P' final states to be very similar in character to those for Be. The heavier alkaline earth atoms $\mathrm{Ca}, \mathrm{Sr}, \mathrm{Ba}$, and $\mathrm{Ra}$ require the treatment of a still larger number of channels due to the proximity in energy of bound " $d$ " orbitals. However, even for these elements, a hyperspherical analysis suggests that the diabatic character of a state populated at small $R$ is preserved as the state evolves toward larger $R[10,28]$. Recently, R-malrix calculations for calcium [31] and strontium [32] have found that at small radial distances the wave functions and channel interactions "look remarkably similar for all of these atoms [ $\mathrm{He}, \mathrm{Be}, \mathrm{Mg}, \mathrm{Ca}, \mathrm{Sr}$ ] including helium" [31a]. In any case, the discovery of the common features of photoexcitation processes in $\mathrm{He}, \mathrm{Be}$, and $\mathrm{Mg}$ as well as in the heavier alkaline carths, despite vast differences in the coupling strength between the associated channels, is one of the new perspectives on twoclcctron correlations provided by the hyperspherical method.

\subsection{Photodelachment of $\mathrm{II}^{-}$}

The first adiabatic hyperspherical calculations for the photodetachment process,

$$
\gamma+\mathrm{H}^{-} \rightarrow \mathrm{H}(n=1)+e^{-},
$$

were carried out independently by Fink and Zoller [33] and by Park et al. [22] and the results agree to within 1\%. Fink and Zoller's results [33] are shown in Fig. 11, where they are compared with results of other authors using different methods [3436], all of which take extensive account of electron correlation effects. As shown in Fig. 11, the adiabatic hyperspherical results are of the order of $10 \%$ higher than the other calculations [34-36] at the peak in the photodetachment cross section and become lower than these other results at energies greater than about 0.1 a.u. above threshold. The decrease in the adiabatic hyperspherical cross section relative to other calculalions at high energies is consistent with what was found in photoionization of He (cf. Fig. 7). Part of the reason for the overestimate of the peak cross section by the adiabatic hyperspherical calculation may be traced to the slight underestimate of the adiabatic hyperspherical value for the $\mathrm{H}^{-}$dissociation energy [37] (i.e., 0.02592 a.u. [38] vs. 0.027751 a.u. [39]). This underestimate causes the adiabatic hyperspherical ground state wave function to be very slightly over-diffuse. This problem, if not addressed, causes severe overestimates of multiphoton detachment cross sections for 


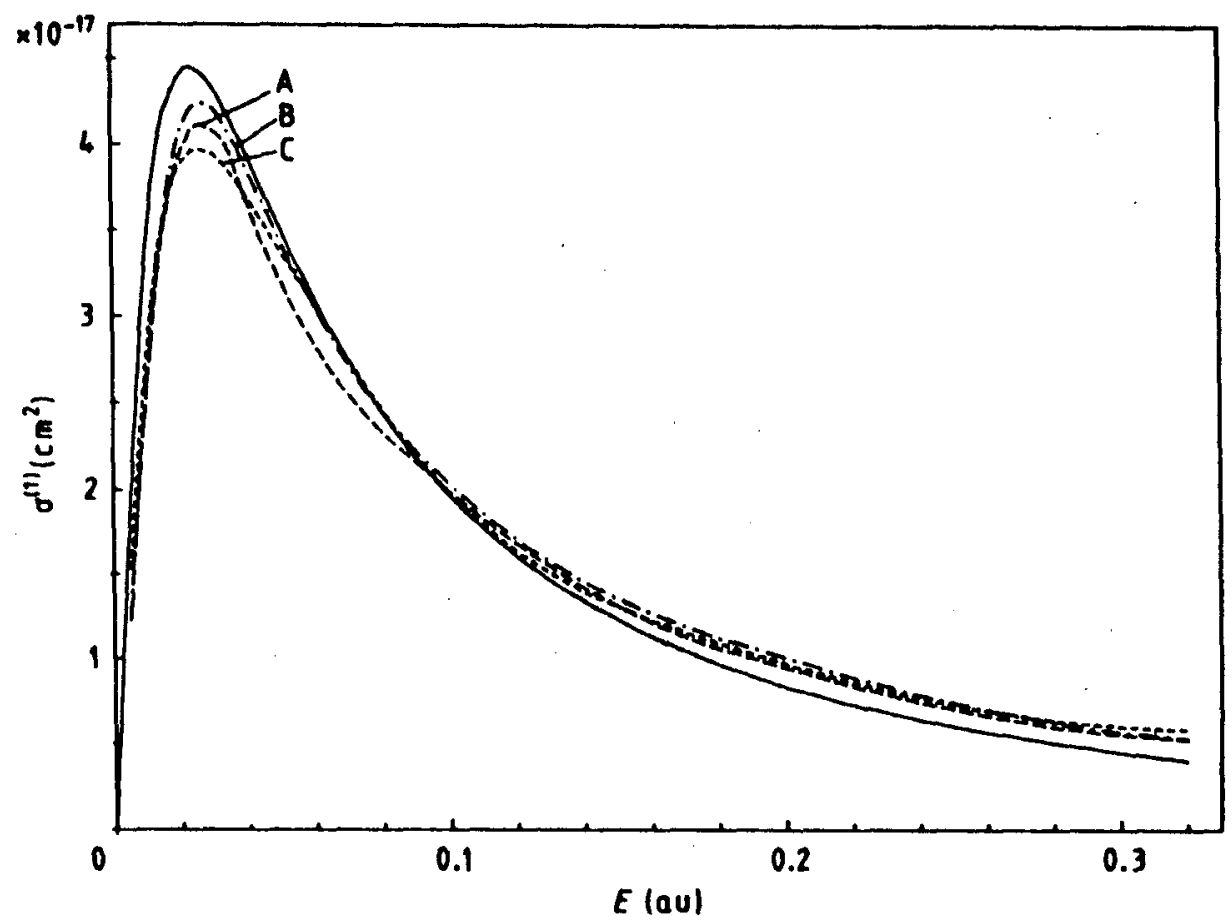

Fig. 11. Photodetachment cross section for $\mathrm{H}^{-}$. Solid Line: Fink and Zoller [33]; A: Daskhan and Ghosh [36]; B: Bell and Kingston [34]; C: Ajmera and Chung [35]. (From Ref. [33].)

$\mathrm{H}^{-}$[37], as demonstrated in the next section. For photodetachment, however, as shown in Fig. 11, the adiabatic hyperspherical results are quite reasonable, considering that they represent a simple, single-channel treatment.

\subsection{Photodetachment of $\mathrm{H}^{-}$with Excitation of $H(n=2)$}

The theoretical description of the photoionization plus excitation process,

$$
\gamma+\mathrm{H}^{-} \rightarrow \mathrm{Il}(n=2)+e^{-},
$$

requires detailed consideration of electron correlations since this process cannot be described by an independent electron model. Liu, Du, and Starace [40] treated process (17) in the adiabatic hyperspherical approximation. Specifically, they calculated the electric dipole transition matrix elements between the ${ }^{1} \mathrm{~S}^{e}$ initial state of $\mathrm{H}^{-}$and the three ${ }^{1} \mathrm{P}^{\circ}$ final states of the $\mathrm{H}(n=2)+e^{-}$system. [The effective potentials corresponding to these three ${ }^{1} \mathrm{P}^{\circ}$ final states are presented in the next section, where we discuss two-photon detachment of $\mathrm{H}^{-}$with excitation of $\mathrm{H}(n=2)$.] Due to the degeneracy of the $H(2 s)$ and $H(2 p)$ levels, there are long-rangle dipole interactions between the detached electron and the $\mathrm{H}(n=2)$ levels. Liu et al. [40] employed the adiabatic hyperspherical representation because it is known [9] to diagonalize these long-rangle dipole interactions. 
The adiabatic hyperspherical results [40] for the total $n=2$ cross section, i.e., $\sigma_{2 s}+\sigma_{2 p}$, are shown in Fig. 12 in comparison with the relative experimental data of Butterfield [41]. As pointed out by Lin [42], the hyperspherical potential ${ }^{1} \mathrm{P}+$ predicts a shape resonance about $18.9 \mathrm{meV}$ too high. In order to compare the $n=$ 2 cross sections with experiment, Liu et al. [40] have shiftedtheir curves $18.9 \mathrm{mcV}$ lower in energy for this figure only. The experimentaldata in Fig. 23 of Ref. [41] have a nonzero background below threshold; this background is subtracted from the data above threshold in Fig. 12. Furthermore, the experimental data have been normalized to the theoretical prediction at the peak of the shape resonance. As shown in Fig. 12, the theoretically predicted $n=2$ cross sertion is dominated by the ${ }^{1} \mathrm{P}+$ shape resonance [43] and is somewhat wider in energy than that measured experimentally. Nevertheless, the agreement is quite reasonable considering that the final-state hyperspherical potentials are uncoupled and, in particular, that there is no coupling to the $\mathrm{H}(n=1)-e^{-1} \mathrm{P}^{\circ}$ channel, indicaling that, according to the calculations of Ref. [40], process (17) is substantially a direct excitation process.

Figure 13 compares the adiabatic hyperspherical results [40] for the total $n=2$ cross section with predictions of Hyman, Jacobs, and Burke [44], Broad and Reinhardt [45], and Wishart [46]. The 1s-2s-2p close-coupling calculation of Hyman, Jacobs, and Burke [44] gives the lowest, broadest, and highest energy prediction for the shape resonance feature. The 160 configuration $J$-matrix calculation of Broad and Reinhardt [45] gives the highest, narrowest, and lowest energy prediction for the shape resonance feature. The hyperspherical results [40] for the height, width, and position of the shape resonance feature are intermediate between the results of these two other calculations. They are close to those of the close-coupling pscudostate plus Hylleraas-type correlation calculation of Wishart [46].

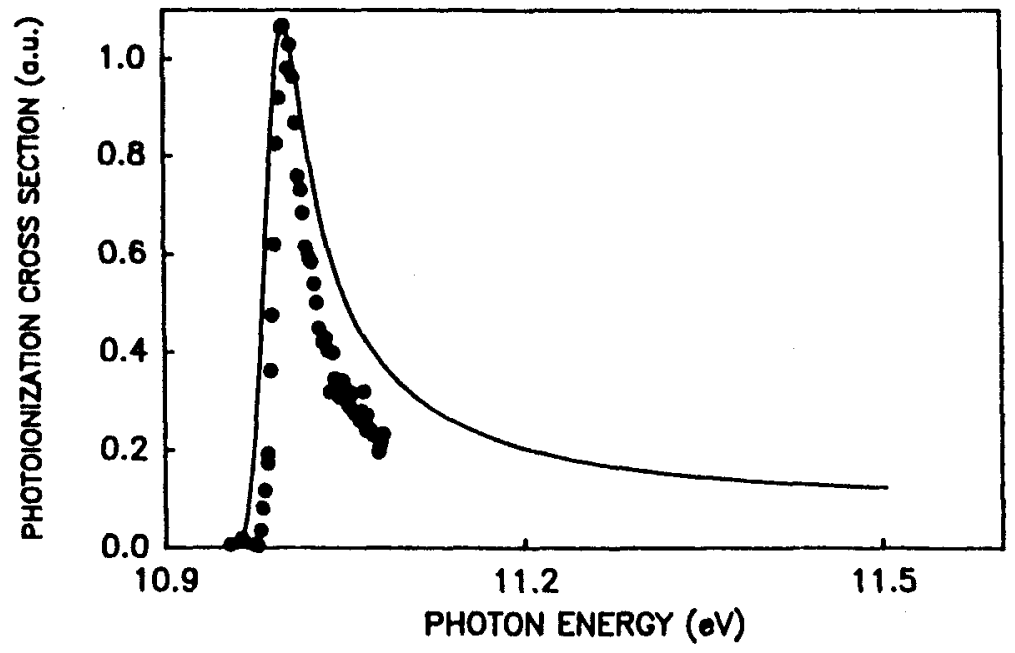

Fig. 12. Photodetachment cross section for the process $\gamma+\mathrm{II}^{-} \rightarrow \mathrm{II}(\mathrm{n}=2)+e^{-}$. Solid line: dipole length adiabatic hyperspherical results of Liu, Du, and Starace [40], shifted to the experimental peak position. Solid circles: relative experimental data of Butterfield (Ref. [41]) normalized to the theoretically predicted peak height. (From Ref. [40].) 


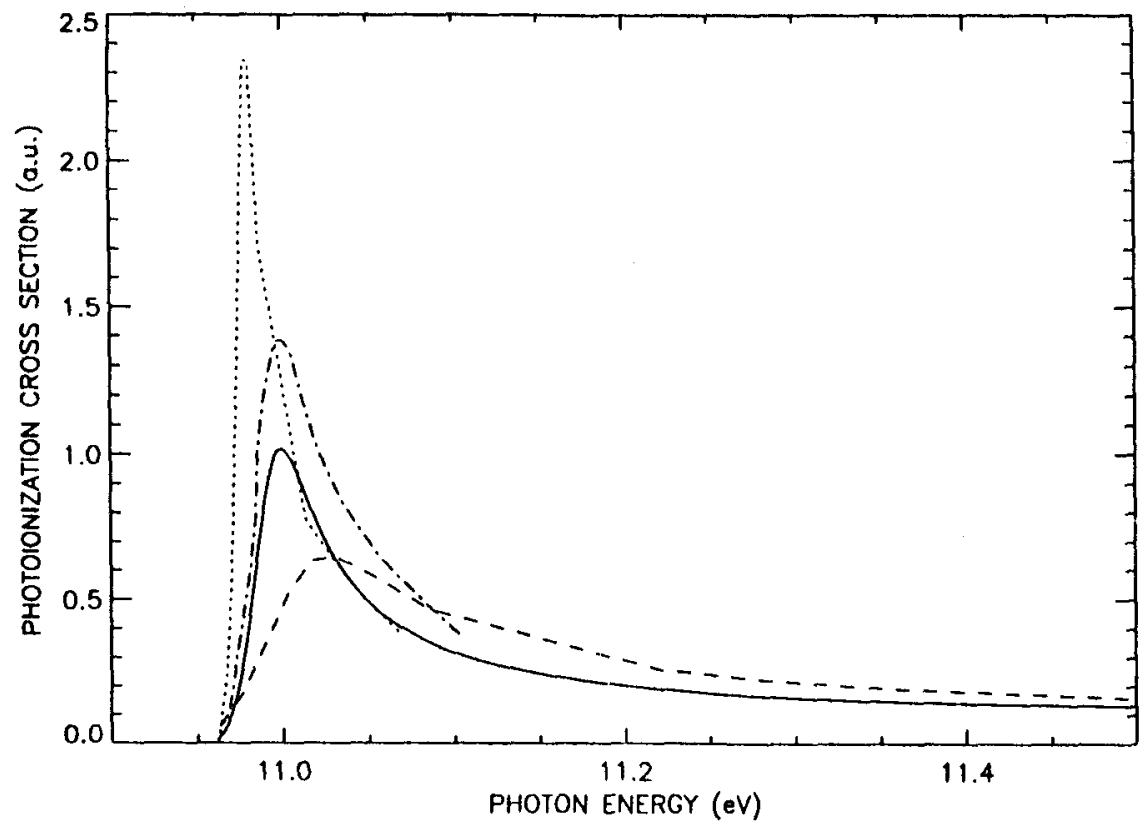

Fig. 13. Theoretical (dipole length) predictions for the photodetachment cross section for the process $\gamma+\mathrm{H}^{-} \rightarrow \mathrm{H}(\mathrm{n}=2)+e^{-}$. Solid line: adiabatic hyperspherical results of Liu, Du, and Starace [40]. Dashed line: 1s-2s-2p close-coupling results of Hyman, Jacobs, and Burke (cf. Table 1 of Ref. [44]). Dotted line: 160 configuration $J$-matrix results of Broad and Reinhardt (cf. Fig. 6 of Ref. [45]). Dot-dashed line: close-coupling pseudostate plus IIylleraas-type correlation calculation of Wishart [46].

The relative experimental results [47] for the total $\mathrm{II}^{-}$detachment cross section in the neighborhood of the $n=2$ threshold have been fitted in detail to the corresponding theoretical results of Broad and Reinhardt [45] taking into account the experimental resolution. Very good agreement was obtained [47]. Nevertheless, the sensitivity of the theoretical calculations for the $n=2$ cross section to the approximations employed, as demonstrated in Fig. 13, indicates a need for an absolute experimental measurement of the $n=2$ cross section.

Liu, Du, and Starace [40] have also presented adiabatic hyperspherical predictions for the $2 p$ and $2 s$ partial cross sections for process (17) as well as for the $2 p$ photoelectron angular distribution asymmetry parameter. The asymmetry parameter agrees very well with that predicted by Hyman, Jacobs, and Burke [44]. The partial cross sections, however, agree more closely with those predicted by Wishart [46].

\subsection{Photodetachment of $I^{-}$with Excitation of $I(n>2)$}

Sadeghpour and Grcene [48] recently calculated the adiabatic hyperspherical potential curves for very highly excited states of $\mathrm{II}^{-}$converging to $\mathrm{H}(n \leq 12)$. Keeping only the lowest " $t$ " states converging to each threshold $H(n)$, they were able to 
interpret the doubly excited resonance structures converging to the $n=4-8$ thresholds that were observed in the photodetachment measurements of Harris et al. [49]. Sadeghpour and Greene [48] interpreted these observed resonances as the doubly excited states supported by the lowest " + " adiabatic hyperspherical radial potentials, as shown in Fig. 14.

The interpretation given by Sadeghpour and Greene [48] for the observed photodetacliment spectra [49] implies that all the other allowed levels supported by the many other adiabatic hyperspherical potentials converging to each $\mathrm{H}(n)$ threshold are not populated in the photodetachment process. Sadeghpour and Greene [48] justified their interpretation by noting that the states corresponding to the lowest + channels converging to each $\mathrm{H}(n)$ threshold have no nodes in the angle $\theta_{12}$ between the two electrons. For example, Fig. 15 shows the adiabatic hyperspherical two-electron density [50] as a contour plot in $0_{12}$ and $\alpha$ for the two lowest + channels converging to the II $(n=6)$ threshold. One can see clearly that the density plot for the lowest + channel in Fig. 15(a) has no $0_{12}$ nodes, whereas that for the next higher + channel in Fig. 15 (b) has a node along $0_{12} \approx 0.75 \pi$. Sadeghpour and Greene [48] therefore

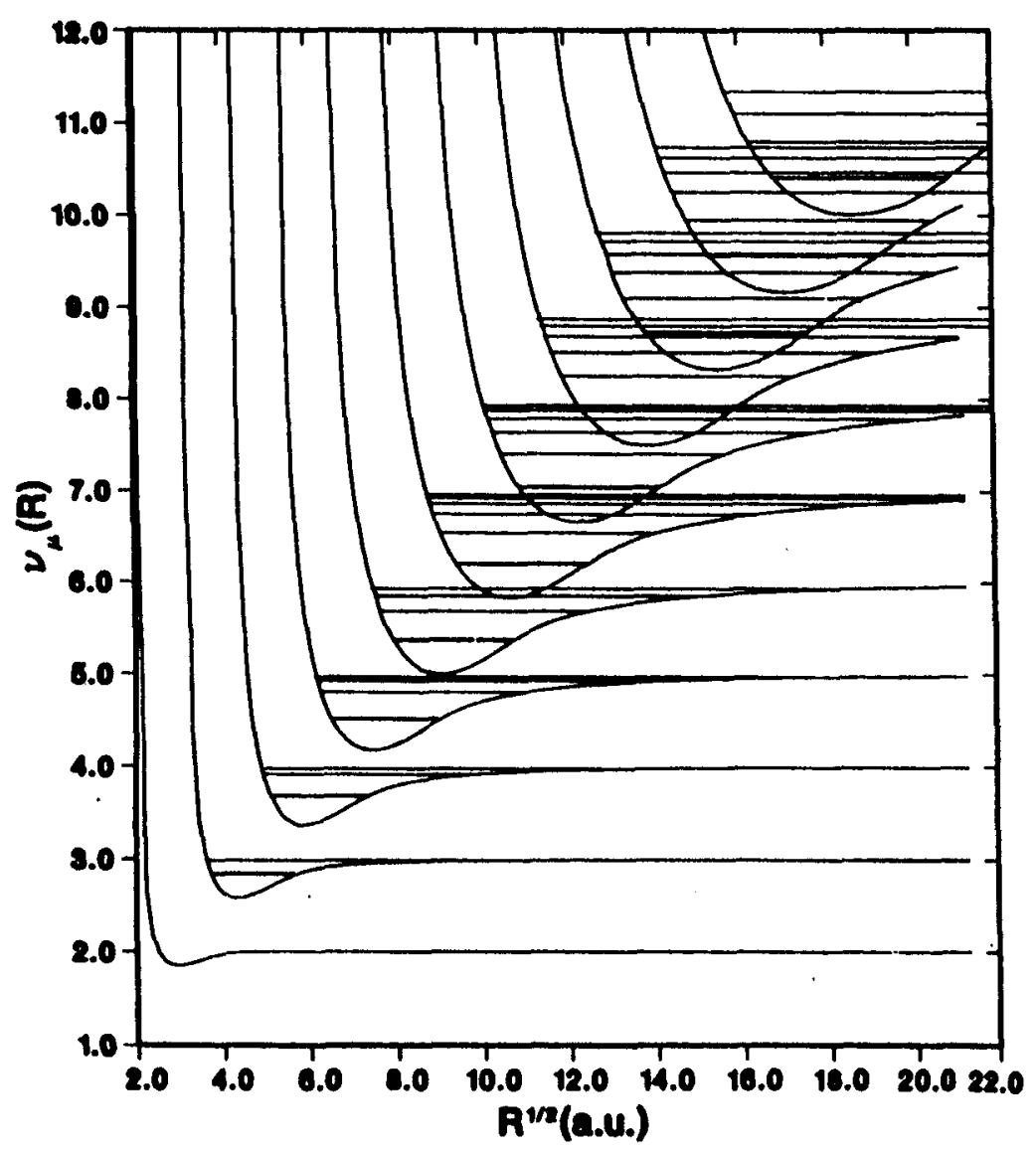

Fig. 14. Adiabatic hyperspherical potentials for the lowest ${ }^{1} \mathrm{Po}^{\circ}+$ channcls of $\mathrm{II}^{-}$plotted as effective quantum numbers $\nu_{\mu}(R) \equiv\left[-2 U_{\mu}(R)\right]^{-1 / 2}$ vs. $R^{1 / 2}$. Doubly exciled state level positions supported within each potential are indicated by horizontal lines. (From Ref. [48].) 

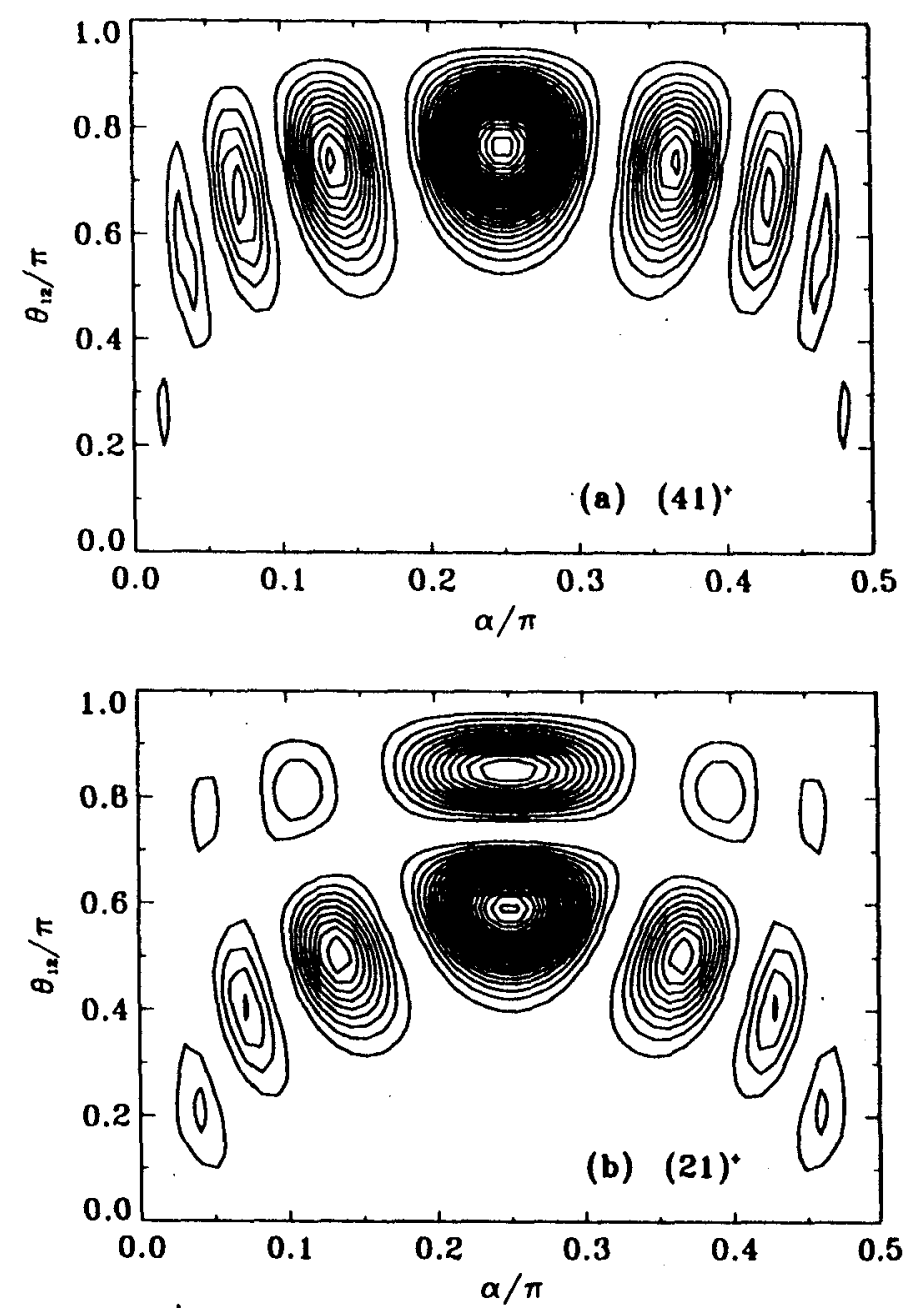

Fig. 15. The adiabatic hyperspherical iwo-electron density function shown as a contour plot vs $\alpha$ and $\theta_{12}$, displaying the nodal patterns for the two lowest + channels in the $n=6$ manifold at $R=80$ a.u. (a) and (b) correspond, respectively, to $(K T)^{A}=(41)^{+}$and $(21)^{+}$ channels, i.e., $v^{A}=0^{+}$and $1^{+}$. (From Ref. [48].)

postulated the propensity rule that in photodetachment of the $\mathrm{H}^{-}$ground state, only doubly excited states having no $\theta_{12}$ nodes are populated with significant intensity.

Sadeghpour and Greene used the bending vibrational quantum number $v$ to quantify the number of nodes in $\theta_{12}$. They postulated that, in general, photoexcitation processes for the ground state of two-electron systems obey the rule, $\Delta v=0$, reasoning that nonadiabatic couplings of transitions from the lowest + channel to higher + channels with $v>0$ are negligible due to the different nodal structures. (Note that Rost, Briggs, and Feagin [51] have pointed out that these nodal structures can be alternatively described in the separable spheroidal coordinates of the molecular orbital picture of two-electron systems $[52,53]$.) 
Very recently, Sadeghpour, Greene, and Cavagnero [54] have carried out eigenchannel R-matrix calculations of the photodetachment cross sections for $\mathrm{H}^{-}$with excitation of the $n=2,3$, and 4 levels of $H$. These calculations give quantitative confirmation of the propensity rules postulated by Ref. [48] on the basis of the adiabatic hyperspherical model.

\subsection{Photoionization of IIe with Excitation of $\mathrm{He}^{+}(n>2)$}

Domke et al. [55] have recently reported the high resolution photoionization study of the doubly excited He states below the $n=2-7$ thresholds of $\mathrm{He}^{+}$. Sadeghpour [56] has shown that the adiabatic hyperspherical representation for highly excited states of He gives a picture similar to that for $\mathrm{H}^{-}$. Namely, the energy levels of doubly excited states calculated in the lowest + adiabatic hyperspherical potentials agree very well with the positions of the experimentally observed [55] resonances for the lowest $n$ levels. Furthermore, the density plots for He display the same kinds of nodal structures, leading to the same conclusion that $\Delta v=0$ is a good propensity rule. For higher $n$ levels, beginning at about $n=6$, overlapping of Rydberg levels corresponding to different $n$ manifolds requires explicit treatment of nonadiabatic coupling terms.

\subsection{Photodetachment of the Positronium Negative Ion}

Botero and Greene [57] have predicted resonant structures in the photodetachment spectrum of $\mathrm{Ps}^{-}$(i.e., $e^{-}-e^{+}-e^{-}$) by using the adiabatic hyperspherical approximation to calculate the relevant adiabatic potential curves. As shown in Fig. 16,

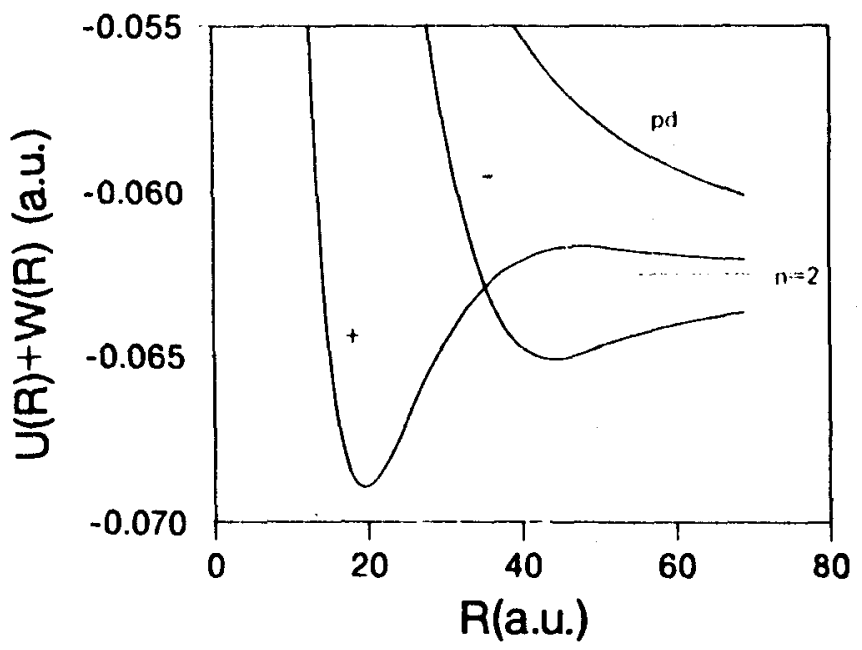

Fig. 16. Hyperspherical potential curves converging to the $\operatorname{Ps}(n=2)$ threshold, including the diagonal adiabatic correction term $W_{\mu \mu}(R)$. These have been interpolated smoothly and diabatically through the avoided crossing at $R=35.5$ a.u. (From Ref. [57].) 
the three potential curves converging to the $n=2$ threshold are very similar to those for $\mathrm{H}^{-}[42]$. The - potential supports an infinite number of Feshbach resonances below the $n=2$ threshold. The + channel does not support any bound resonances, but has a potential barricr which should produce a shape reasonance above threshold. Botero and Grecne [57] predict this shape resonance to lie $4 \times 10^{-4}$ a.u. above the $n=2$ threshold. As yet, there are no experimental measurements for this photodetachment process, but the existence of the shape resonance has been verified by the molecular orbital model theory of threc-body Coulomb systems of Feagin and Briggs $[52]$.

\section{Description of Multiphoton Processes}

\subsection{Multiphoton Transition Matrix Elements}

Consider first the transition amplitude for a two-photon transition from an initial statc $\mid i>$ to a final statc $<f \mid$ :

$$
T_{i \rightarrow f}^{(2)}=\left\langle f\left|D \frac{1}{E_{i}+\omega-H} D\right| i\right\rangle,
$$

where the electric dipole operator $D$ is defined by $\mathrm{Eq}$. (13) (for linearly polarized light) and where the atomic Hamiltonian is defined implicitly by Eq. (3). One way to evaluate Eq. (18), first introduced by Fink and Zoller [33], is to employ the DalgarnoLewis [58] procedure in the adiabatic hyperspherical representation. In this method one introduces the ket $|\lambda\rangle$,

$$
|\lambda\rangle \equiv \frac{1}{E_{i}+\omega-H} D|i\rangle
$$

which may be obtained numcrically by solving the inhomogeneous equation

$$
\left(E_{i}+\omega-H\right)|\lambda\rangle=D|i\rangle \text {. }
$$

Having obtaincd the ket $|\lambda\rangle$, the two-photon transition amplitude is given by

$$
T_{i \rightarrow f}^{(2)}=\langle f|D| \lambda\rangle \text {. }
$$

An alternative procedure is to use the variationally stable form for multiphoton transition malrix clcments introduced by Gao and Starace [59]. In this procedure, one introduces in addition to the ket $|\lambda\rangle$ in Eq. (19) the bra $\left\langle\lambda^{\prime}\right|$, defined formally by

$$
\left\langle\lambda^{\prime}\right\rangle \equiv\langle f| D \frac{1}{E_{i}+\omega-I I}
$$

The variationally stable form for the two-photon transition matrix element is then given by [59]:

$$
T_{i \rightarrow f}^{(2)}=\langle f|D| \lambda\rangle+\left\langle\lambda^{\prime}|D| i\right\rangle-\left\langle\lambda^{\prime}\left|E_{i}+\omega-H\right| \lambda\right\rangle \text {. }
$$


In Eq. (23), cach of the three matrix elcments are in principle equal to each other. (The equality of the third term to the second term, for example, may be seen by using Eq. (20) to replace $D|i\rangle$ in the sccond term.) Equation (23) is variationally stable in the sense that any errors in the determination of $|\lambda\rangle$ and $\left(\lambda^{\prime} \mid\right.$ enter Eq. (23) only in quadratic and higher powers; no linear terms in these errors appear [59].

The evaluation of Eq. (23) in the adiabatic hyperspherical approximation proceeds as follows (and the corresponding evaluation of Eq. (21) will be noted as a special case): We express the initial, final, and intermediate states in terms of adiabatic hyperspherical wave funclions:

$$
\begin{aligned}
|i\rangle & =\left(R^{5 / 2} \sin \alpha \cos \alpha\right)^{-1} F_{\mu_{i}}(R) \phi_{\mu_{i}} \\
|f\rangle & =\left(R^{5 / 2} \sin \alpha \cos \alpha\right)^{-1} F_{\mu_{f}}(R) \phi_{\mu_{j}} \\
|\lambda\rangle & =\left(R^{5 / 2} \sin \alpha \cos \alpha\right)^{-1} \lambda_{\mu}(R) \phi_{\mu} \\
\left|\lambda^{\prime}\right\rangle & =\left(R^{5 / 2} \sin \alpha \cos \alpha\right)^{-1} \lambda_{\mu}^{\prime}(R) \phi_{\mu}
\end{aligned}
$$

The first two matrix elements in Eq. (23) thus become:

$$
\begin{aligned}
& \left\langle\lambda^{\prime}|D| i\right\rangle=\int_{0}^{\infty} I_{\mu \mu_{i}}^{L}(R) \lambda_{\mu}^{\prime}(R) R F_{\mu_{i}}(R) d R \\
& \langle\digamma|D| \lambda\rangle=\int_{0}^{\infty} I_{\mu_{\mu} \mu}^{L}(R) F_{\mu j}(R) R \lambda_{\mu}(R) d R,
\end{aligned}
$$

where the angular integrals $I_{\mu^{\prime} \mu}^{L}(R)$ have been given in general by Park et al. [22] and have been given for the particular case of ${ }^{1} \mathrm{~S} \rightarrow{ }^{1} \mathrm{P}$ transitions in Eq. (14) above. The third matrix element in Eq. (23) becomes

$$
\left\langle\lambda^{\prime}\left|E_{i}+\omega-H\right| \lambda\right\rangle=\left\langle\lambda_{\mu}^{\prime}(R)\left|E_{i}+\omega-h_{\mu}^{\text {ad }}(R)\right| \lambda_{\mu}(R)\right\rangle
$$

where

$$
h_{\mu}^{a d}(R) \equiv-\frac{1}{2}\left[\frac{d^{2}}{d R^{2}}+\frac{U_{\mu}(R)+\frac{1}{4}}{R^{2}}+\left(\phi_{\mu}, \frac{\partial^{2}}{\partial R^{2}} \phi_{\mu}\right)\right] .
$$

The two numcrical procedures for obtaining the two-photon transition element in Eq. (18) in the adiabatic hyperspherical method are thus as follows. In the DalgarnoLewis procedurc [58], Eq. (21) is cvaluated using Eq. (29) in which the radial function $\lambda_{\mu}(R)$ is obtained by solving the radial equivalent of the inhomogeneous Eq. (20). In contrast, in the variationally stable procedure, the three terms of Eq. (23) are cvaluated using Eqs. (28), (29), and (30). The functions $\lambda_{\mu}(R)$ and $\lambda_{\mu}^{\prime}(R)$ are cach expanded in an $L^{2}$ basis of Slater orbitals. The coefficients of this expansion are determined by requiring that Eq. (23) be variationally stable [59].

Generalization of these two methods to calculate perturbative N-photon transition matrix clements is straightforward. In particular, variationally stable expressions for three-photon transition matrix elements in the adiabatic hyperspherical representation have been presented explicitly $[60,37]$. 


\subsection{Multiphoton Delachment of $\mathrm{H}^{-}$}

Fink and Zoller [33] presented the first adiabatic hyperspherical results for twophoton detachment of $\mathrm{HI}^{-}$. Their calculations were limited to the case of circularly polarized light. As documented by Geltman [61], the current status of the theory for multiphoton detachment of $\mathrm{H}^{-}$is rather confused, with results of different groups differing in magnitude by factors of 2 or so. In particular, as shown in Fig. 6 of Ref. [61], the circularly polarized results of Fink and 7oller [33] for two-photon detachment of II $^{-}$appear to be on the low side as compared to results of other groups.

Forthcoming results of Liu, Gao, and Starace [37] shed some light on some causes of the difficulty in obtaining accurate magnitudes for the multiphoton detachment cross sections of $\mathrm{II}^{-}$. A key cause is the sensitivity of the cross sections to the $\mathrm{H}^{-}$ dissociation encrgy. One may cstimate analytically that any crrors in the dissociation energy lead to errors in the $\mathrm{N}$-photon detachment cross section that are roughly proportional to the factor $(4 N-1)$. (The magnitude of the error will of course diminish as the photoclectron's kinetic energy increases.) Thus, the errors in the multiphoton cross sections can become very large with increasing $N$.

Liu, Gao, and Starace [37] addressed this problem by semi-empirically adjusting their ground state adiabatic hyperspherical potential for $\mathrm{H}^{-}$so that the ground state energy was in agrecment with experiment. This change resulted in a $25 \%$ reduction of the peak valuc of the two-photon detachment cross section and a $40 \%$ reduction in the peak value of the threc-photon cross section, as shown in Fig. 17.

The results including semi-empirical adjustments now lie much closer to accurate, short-range potential model results $[61,37]$. The remaining differences probably result from electron correlation effects, which are included in the adiabatic hyperspherical calculations [37].

\subsection{Two-Photon Detachment of $I^{-}$with Excitation of $H(n=2)$}

We shall be concerned here with the two-photon detachment of the hydrogen negative ion accompanicd by the simultaneous excitation of the resulting $\mathrm{H}$ atom to its $n=2$ state, i.c.,

$$
\mathrm{II}^{-}+2 \gamma \rightarrow \mathrm{H}(n=2)+e^{-} .
$$

Due to the degencracy of the $11(2 s)$ and $\mathrm{H}(2 \mathrm{p})$ states, the final state of this process is influenced by the long-range dipole field interaction between the $\mathrm{H}$ atom and the detached electron. Liu, Du, and Starace [40] have shown, using adiabatic hyperspherical and quantum defect theory methods, that this process is probably the most favorable one for obscrving dipole-ficld-induced oscillations in the cross sections near threshold. Such oscillations were predicted long ago by Gailitis and Damburg [62], but have never been observed experimentally. Before presenting the results of Ref. [40], we discuss first the kcy aspects of the long-range dipole field interactions for process (32). 

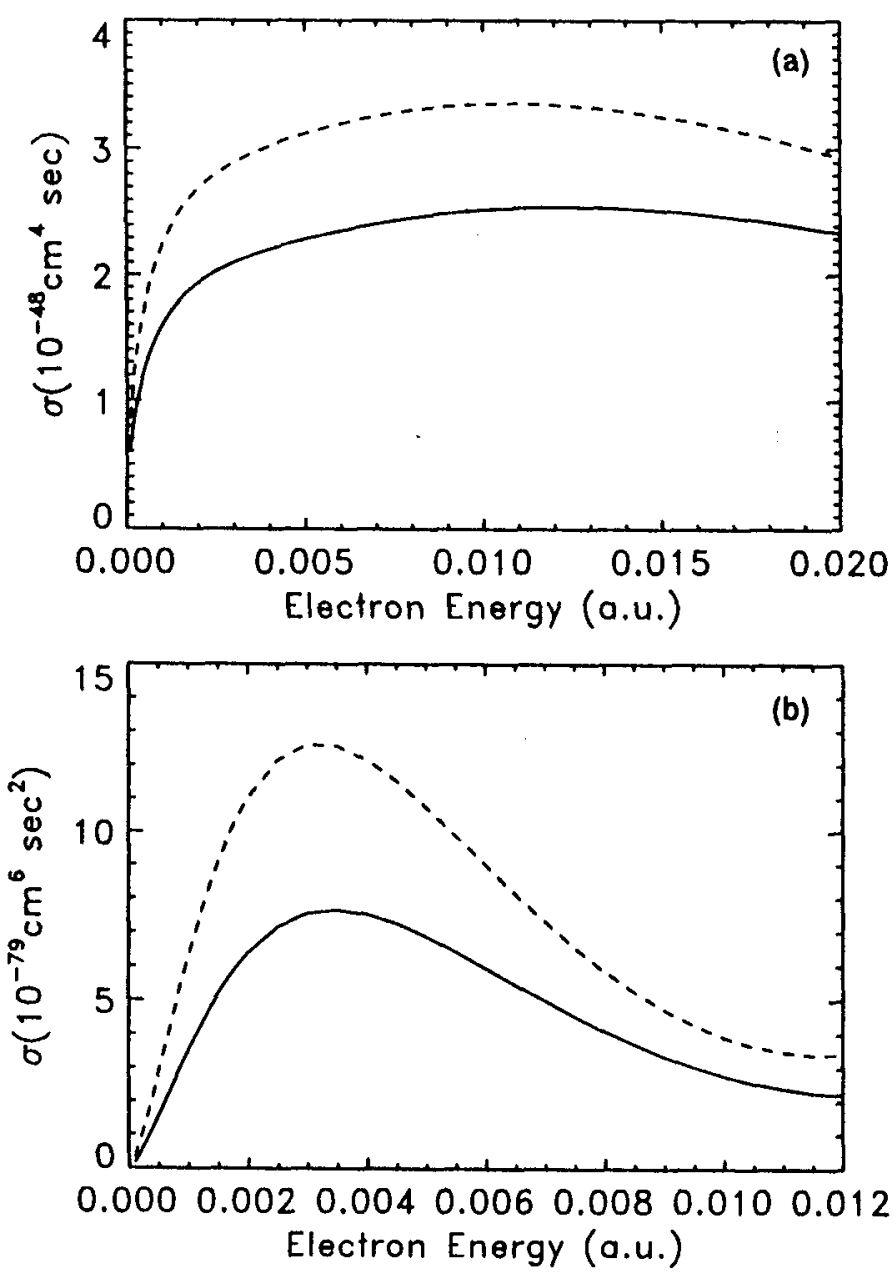

Fig. 17. Variationally stable, adiabatic hyperspherical cross section results for (a) two- and (b) three-photon detachment of $\mathrm{H}^{-}$. Solid lines: with semi-empirical ground state potential adjustment. Dashed lines: without semiempirical adjustment. (From Ref. [37].)

4.3.1. Low-Energy States of the $H(n=2)-e^{-}$System. In the adiabatic hyperspherical representation, the radial channel functions $F_{\mu}(R)$ satisfy the one-dimensional radial Schrödinger equation,

$$
\left[\frac{d^{2}}{d R^{2}}-V_{\mu}(R)+k^{2}\right] F_{\mu k}(R)=0,
$$

where $k$ is the asymptotic value of the momentum of the detached electron and where the effective radial potential $V_{\mu}(R)$, which characterizes the dynamical features of a particular hyperspherical channel $\mu$ converging to the nth level of the II atom, is 
defined by

$$
-V_{\mu}(R) \equiv \frac{U_{\mu}(R)+\frac{1}{4}}{R^{2}}+\left(\phi_{\mu} \frac{\partial^{2} \phi_{\mu}}{\partial R^{2}}\right)-\frac{1}{n^{2}} .
$$

Since the long-range dipole interaction due to the degeneracy of the $\mathrm{H}(\mathrm{n}=2)$ states [63] is diagonal in the hyperspherical representation $[9,42]$, the asymptotic form of the effective radial potential is

$$
V_{\mu}(R) \underset{R \sim \infty}{\sim} \lambda_{\mu}\left(\lambda_{\mu}+1\right) / R^{2} .
$$

In Eq. (35) $\lambda_{\mu}$ is an effective orbital angular momentum, which may be real or complex depending on the channel $\mu$. For channels $\mu$ in which the long-rangle dipole interaction [63] is repulsive at asymptotic distances, $\lambda_{\mu}$ is real. Hence at threshold the cross section for any excitation to the channel $\mu$ is zero since it depends on $\left|k^{\lambda_{\mu}+1 / 2}\right|^{2}$, which is zero for $k \rightarrow 0$. On the other hand, for channels $\mu$ in which the long-range dipole interaction [63] is attractive at asymptotic distances, one may write quite generally [64],

$$
\lambda_{\mu}=-\frac{1}{2}+i \alpha_{\mu} .
$$

As a consequence, the threshold value of the cross section for any excitation to the channel $\mu$ is finite [62] since it depends on $\left|k^{\lambda_{\mu}+1 / 2}\right|^{2}=1$. In addition, as noted by Gailitis and Damburg [62], the transition matrix elements for channels having complex $\lambda_{\mu}$ are influenced above threshold by the term $k^{\lambda_{\mu}+1 / 2}=k^{i \alpha_{\mu}}$ (cf. Eq. 36), which, when rewritten as $\exp \left(i \alpha_{\mu} \ln k\right)$, may be seen to osciliate as a function of $\ln k$.

Some of the effective potentials $V_{\mu}(R)$ which converge asymptotically to the $\mathrm{H}(\mathrm{n}=2)$ threshold are shown in Fig. 18. All of the ${ }^{1} \mathrm{~S}^{e}$ and ${ }^{1} \mathrm{P}^{\circ}$ potential curves are shown as well as the most important ${ }^{1} D^{e}$ potential curve. Since the total orbital and spin angular momenta are insufficient to specify the potential curves uniquely, additional

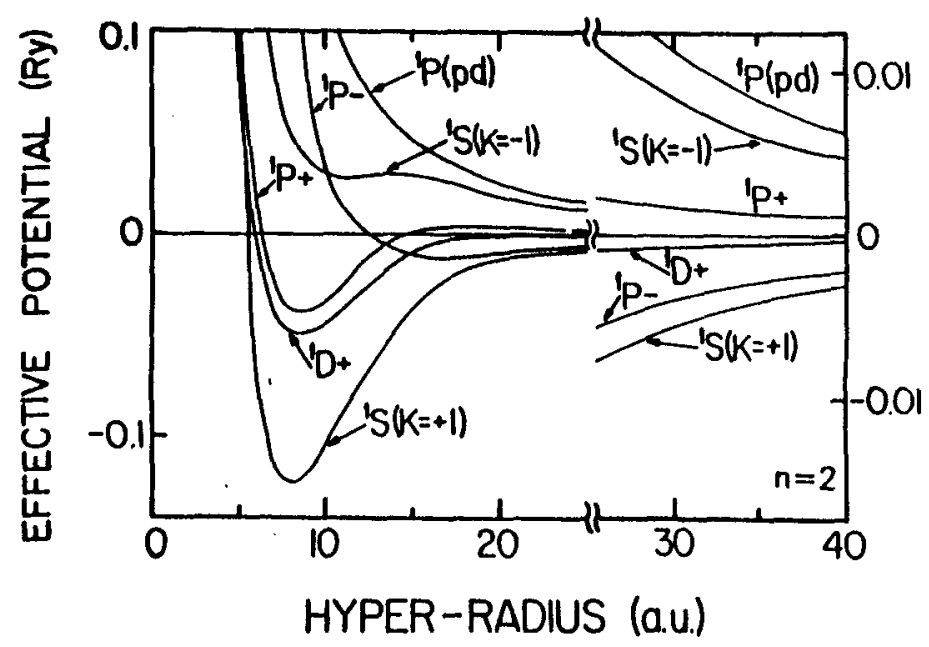

Fig. 18. Effective radial hyperspherical potentials $V_{\mu}$ in Ry plotted vs the hyperradius $R$ for six channels converging to II $(\mathrm{n}=2):{ }^{1} \mathrm{~S}(\mathrm{~K}= \pm 1),{ }^{1} \mathrm{P} \pm,{ }^{1} \mathrm{P}(\mathrm{pd})$, and ${ }^{1} \mathrm{D}+$. Note that the zero of energy is chosen to be the $\mathrm{II}(\mathrm{n}=2)$ threshold and that near $R=25$ the vertical energy scale is changed. (From Ref. [40].) 
specification is necessary. In Fig. 18 we have employed abbreviated labels corresponding to Lin's classification of doubly excited states [65].

The key features of the interactions within the $\mathrm{H}(\mathrm{n}=2)-e^{-}$system are clearly exhibited in the effective polential curves shown in Fig. 18. These are, first, that the ${ }^{1} \mathrm{P}+$ potential is attractive at short distances and weakly repulsive at large distances thereby giving rise to a shape resonance [42] (which is seen experimentally at about 18 $\mathrm{meV}$ above threshold). This shape resonance feature dominates the cross section of any process which populates the ${ }^{1} \mathrm{Po}^{\circ}$ final state channels above the $\mathrm{H}(\mathrm{n}=2)$ threshold (cf. Fig. 12).

Second, because of their long-range repulsive behavior, the ${ }^{1} \mathrm{P}+,{ }^{1} \mathrm{P}(\mathrm{pd})$, and ${ }^{1} \mathrm{~S}(\mathrm{~K}=-1)$ potentials all have zero cross sections at threshold.

Third, the three potentials corresponding to the ${ }^{1} \mathrm{~S}(\mathrm{~K}=+1),{ }^{1} \mathrm{P}-$, and ${ }^{1} \mathrm{D}+$ channcls are altractive at asymptotic distances. As discussed above, they therefore have complex effective angular momenta. Ilence the excitation cross section for each of these channcls is finile at threshold (within the center-of-mass frame of the $\mathrm{H}(\mathrm{n}=2)$ $-e^{-}$system). Furthermore, the transition amplitudes for excitations to these three channels having complex effective angular momenta oscillate on a $\ln k$ scale above threshold [62].

4.3.2. Key Aspects of the Two-Photon Detachment Process. The two-photon detachment process in Eq. (32) is a very favorable one for observing Gailitis-Damburg oscillations [62] above the $\mathrm{II}(\mathrm{n}=2)$ threshold [40]. This is so for two reasons. First, electric dipole selection rules do not permit population of ${ }^{1} \mathrm{P}^{\circ}$ final state channels. Hence the strong shape resonance in the ${ }^{1} \mathrm{P}+$ final state channel about $18 \mathrm{meV}$ above threshold cannot obscure these near-threshold oscillations. Second, the two-photon process does populate ${ }^{1} \mathrm{~S}^{e}$ and ${ }^{1} \mathrm{D}^{e}$ final state channels, one of which, the ${ }^{1} \mathrm{D}+$ channel, is the only one with significant, undamped oscillations above threshold [40].

Before demonstrating these Gailitis-Damburg oscillations [62] for this process one must ask how one can be sure that the wiggles that the calculations give for the two-photon detachment plus excitation cross sections of $\mathrm{II}^{-}$are really due to longrange dipolc field effects and are not due to some other cause. The answer is that the generalized quantum defect theory (QD'T) of Greene, Fano, and Strinati [64] for a long range dipole ficld enables one to disentangle dipole-field effects from our numerical results analytically. In this way one is able to state with assurance which features of the cross section results are truly the Gailitis-Damburg oscillations [62] and which features are energy-dependent wiggles arising from other causes.

Through use of the QDT for long-range dipole fields [64], one may show that the adiabatic hyperspherical radial functions, defined by Eq. (33), tend asymptotically to

$$
F_{\mu k}(r) \underset{R \sim \infty}{\sim}(2 / \pi k)^{1 / 2} \sin \left(k R+\xi_{\mu}+\eta_{\mu}\right),
$$

where $\eta_{\mu}$ is the phase shift in the $\mu$ th cliannel and $\xi_{\mu}$ is an analytic phase dependent on the effective angular momentum $\lambda_{\mu}$ characterizing the long-range dipole interaction of the $\mathrm{H}(\mathrm{n}=2)-e^{-}$system [64] in this channel. For real values of $\lambda_{\mu}$,

$$
\xi_{\mu} \equiv-\frac{1}{2} \pi \lambda_{\mu}
$$


while for complex values of $\lambda_{\mu}[64]$,

$$
\xi_{\mu} \equiv-\frac{1}{4} \pi+\theta_{\mu}
$$

where (cf. Eq. 8)

$$
\theta_{\mu} \equiv-\tan ^{-1} \frac{\tan \left[\alpha_{\mu} \ln (k / 2)+x_{\mu}\right]}{\tanh \left(\pi \alpha_{\mu} / 2\right)}
$$

and

$$
x_{\mu} \equiv \arg \Gamma\left(1-i \alpha_{\mu}\right) \text {. }
$$

The generalized QDT may also be used to extract the long-range dipole-field-induced energy dependence of $F_{\mu k}(R)$ by representing the adiabatic hyperspherical radial wave functions as [66]

$$
F_{\mu k}(R)=N_{\mu}(k) F_{\mu k}^{\circ}(R)
$$

where $N_{\mu}(k)$ is an effective normalization factor which determines essentially all of the energy dependence of the radial wave function near $R \approx 0$, and where $F_{\mu k}^{\circ}(R)$ is a more smoothly varying function of $k$. The oscillatory, energy-dependent normalization factor $N_{\mu}(k)$ is an analytically known function of $\ln k[40,66]$.

There are two ways in which an attractive dipole field introduces oscillations in measured cross sections on a $\ln k$ energy scale. The first is due to the rapid variation of the analytically determined dipole phase $\theta_{\mu}$ (cf. Eqs. 39 and 40) for those hyperspherical channels $\mu$ having complex values of the effective angular momentum $\lambda_{\mu}$. This analytically determined phase $\theta_{\mu}$ (through $\xi_{\mu}$ ) appears explicitly in the phase factor included in the two-photon transition amplitudes [40]. Interference effects between different amplitudes, such as occur commonly in calculating the angular distributions for the detached electrons, generally lead to sizable, undamped oscillations in the corresponding cross sections due to the rapid decrease of the analytically determined phases $\theta_{\mu}$ with increasing $\ln k$. This analytic behavior is shown in Fig. 19 for all three channels having complex $\lambda_{\mu}$ above the $\mathrm{H}(\mathrm{n}=2)$ threshold.

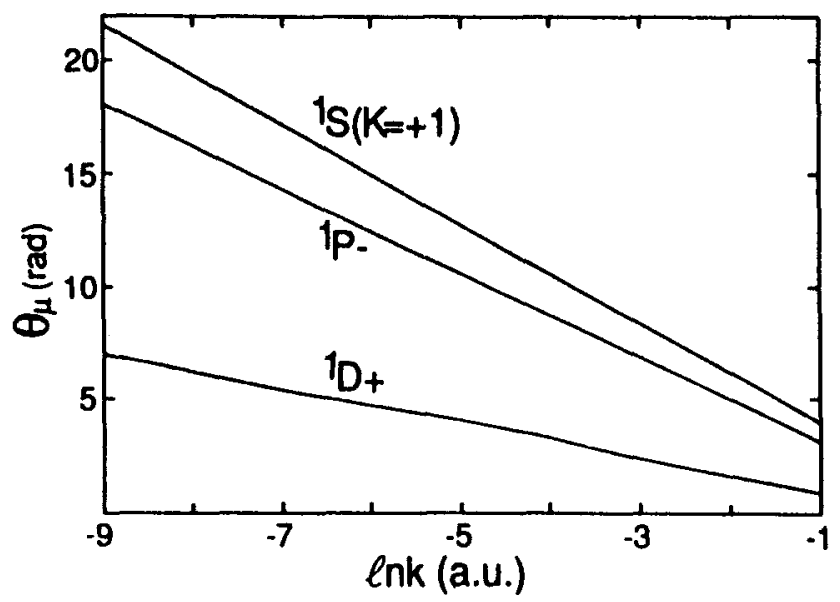

Fig. 19. Analytic phase $\theta_{\mu}$ (defined in Eq. 40) vs. In $k$, where $k$ (a.u.) is the detachedelectron momentum, for the three adiabatic hyperspherical channels $\mu={ }^{1} \mathrm{~S}(\mathrm{~K}=+1),{ }^{1} \mathrm{P}-$, and ${ }^{1}$ Dt. (From C. R. Liu and A. F. Starace, Phys. Rev. A 40 (1989) 4926.) 
The second way the long-range dipole field introduces oscillations in the cross sections is through the effective normalization $N_{\mu}(k)$ introduced in Eq. (42). Its behavior is shown in Fig. 20 for each of the three channels above the $\mathrm{H}(\mathrm{n}=2)$ threshold having complex $\lambda_{\mu}$. One sees clearly that whereas the long-range dipole-field-induced oscillations of $N_{\mu}(k)$ for the ${ }^{1} \mathrm{~S}(\mathrm{~K}=+1)$ and ${ }^{1} \mathrm{P}$ - channels are strongly damped, those for the ${ }^{1} \mathrm{D}+$ channel are quite sizable [40].

4.3.3. Results. The total cross sections for the two-photon detachment of $\mathrm{H}^{-}$with excitation of $\mathrm{H}(\mathrm{n}=2)$ (cf. Eq. 32) are given for the cases of linearly (L) and circularly (C) polarized light in Figs. 21(a) and 21(b) respectively. In Figs. 21(c) and 21(d) the generalized QDT $[64,66]$ is used to extract analytically the energy-dependence arising from the long-range dipole field in order to give renormalized cross sections [40]. One sees clearly that for electron momenta such that $\ln k \lesssim-3.0$, the oscillations of the cross sections are due to the long-range dipole field. Now, for $\ln k \lesssim-6.0$, the assumed degeneracy of $\mathrm{H}(2 \mathrm{~s})$ and $\mathrm{H}(2 \mathrm{p})$ breaks down due to spin-orbit and Lamb shift effects. Thus, for $-6.0 \lesssim \ln k \lesssim-3.0$ or, alternatively, for detached electron kinetic energies from $\approx 0.1 \mathrm{meV}$ to $\approx 34 \mathrm{neV}$, the energy dependence of the cross sections may be ascribed to Gailitis-Damburg oscillations [62]. As shown in Figs. 21 (a) and 21(b), this energy region corresponds to a half-cycle of such oscillation over which the $\mathrm{L}$ cross section increases by $\approx 30 \%$ and the $\mathrm{C}$ cross section increases by $\approx 50 \%$.

Figure 22 presents results [40] for the total $n=2$ differential cross section, which is the sum of the differential cross sections for the $2 \mathrm{~s}$ and $2 \mathrm{p}$ states. Results are given for the six angles, $\theta_{k}=0^{\circ}, 18^{\circ}, 36^{\circ}, 54.7^{\circ}, 72^{\circ}$, and $90^{\circ}$. [Note that the results labelled $\theta_{k}=54.7^{\circ}$ are actually calculated for $\theta_{k}=54.7356^{\circ}$, at which $P_{2}\left(\theta_{k}\right)=0$.] One sees from this figure that the energy dependence of the differential cross section in the region $-6 \lesssim \ln k \lesssim-3$ (over which long-range dipole field effects play the major role)

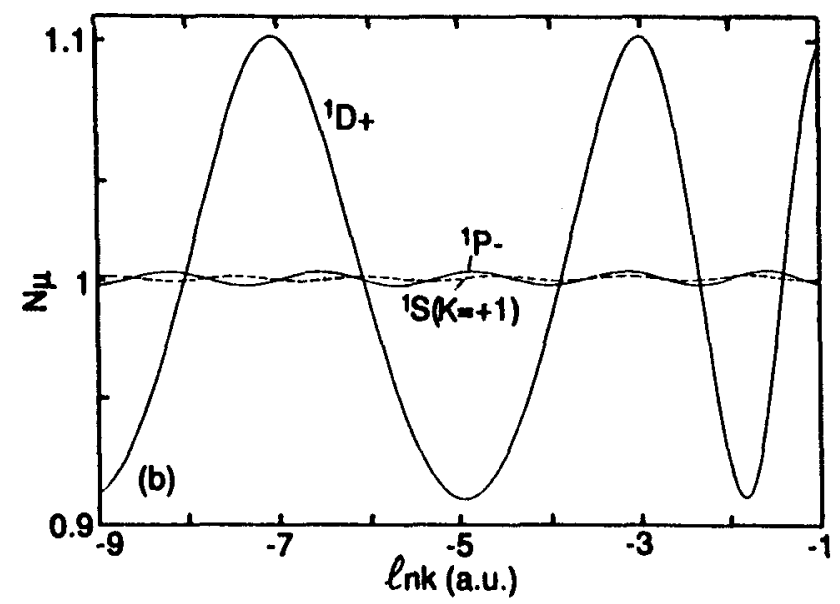

Fig. 20. Normalization factors $N_{\mu}(k)$ (cf. Eq. 42) for the three adiabatic hyperspherical channels $\mu={ }^{1} \mathrm{~S}(\mathrm{~K}=+1),{ }^{1} \mathrm{P}-$, and ${ }^{1} \mathrm{D}+$ vs. In $k$, where $k$ is the detached-electron momentum. (From C. R. Liu and A. F. Starace, Phys. Rev. A 40 (1989) 4926.) 

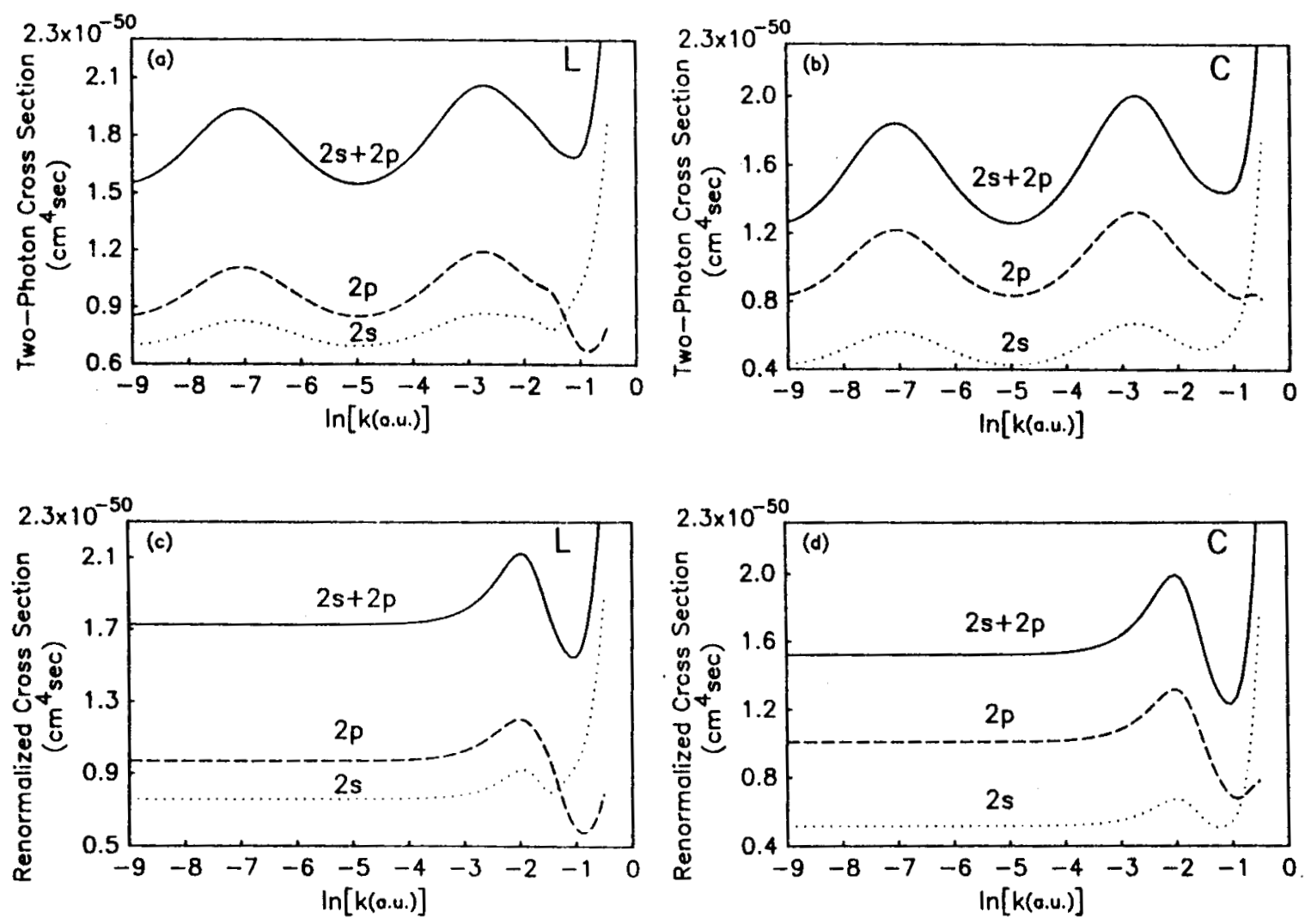

Fig. 21. Generalized two-photon cross sections for the cases of linearly (L) and circularly (C) polarized light for the process $2 \gamma+\mathrm{H}^{-} \rightarrow \mathrm{H}(2 \mathrm{~s}, 2 \mathrm{p})+e^{-}$plotted vs. In $k$, where $k$ (a.u.) is the photoelectron momentum. (a) L results; (b) C results; (c) renormalized L results; (d) renormalized $\mathrm{C}$ results. (From Ref. [40].)
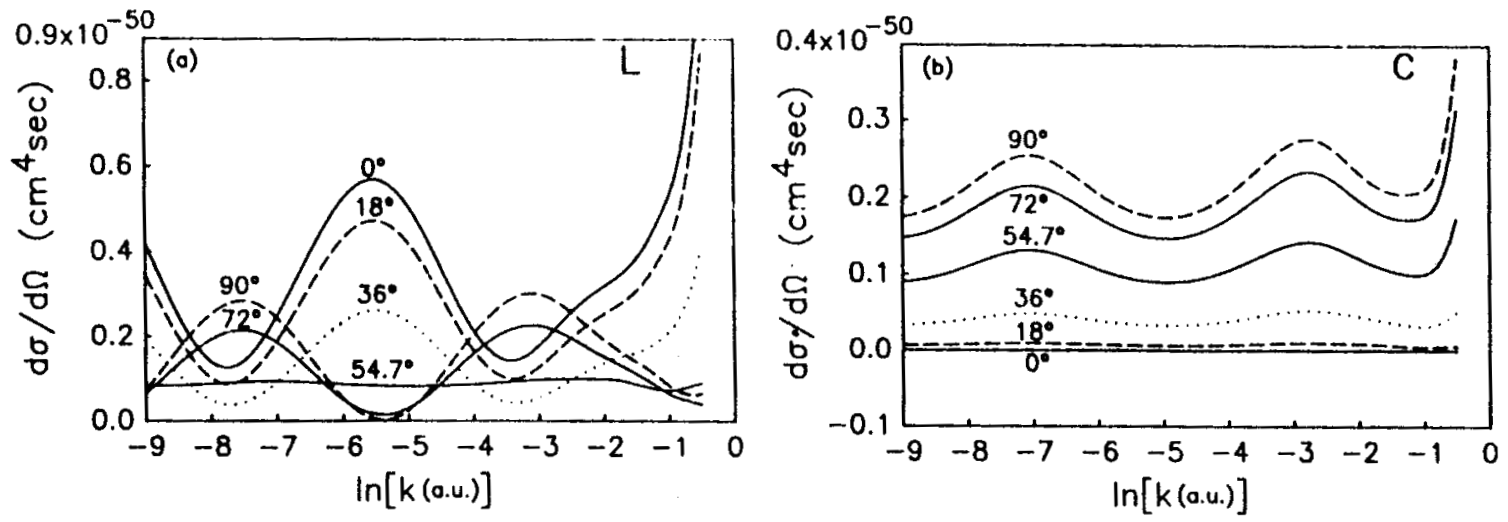

Fig. 22. Differential cross section for the cases of linearly $L$ and circularly (C) polarized light for the process $2 \gamma+\mathrm{II}^{-} \rightarrow \mathrm{H}(\mathrm{n}=2)+e^{-}$plotted vs. $\ln k$, where $k$ (a.u.) is the photoelectron momentum, for the detached electron angles $\theta_{k}=0^{\circ}, 18^{\circ}, 36^{\circ}, 54.7356^{\circ}, 72^{\circ}$, and $90^{\circ}$. (a) L results; (b) C results. (From Ref. [40].) 
is highly dependent on the angle $\theta_{k}$ at which the photoelectron is detected. This energy dependence may be enhanced by use of linearly polarized light and small angles of detection, $\theta_{k}$.

Note that the cnergy dependences of the total two-photon detachment cross sections prescnted in Fig. 21 are governed primarily by the long-range dipole field normalization factors $N_{\mu}(k)$ (cf. Fig. 20). The differential cross sections in Fig. 22 are strongly influenced in addition by the rapidly decreasing analytic phases $\theta_{\mu}$ (cf. Fig. 19). Indeed the encrgy-dependence of the asymmetry parameters for the two-photon process is primarily governed by these analytic phases. Thus, the long-range dipole ficld effects due primarily to $N_{\mu}(k)$ can be found by measuring the total cross sections, while those due primarily to $\theta_{\mu}$ can be found by measuring the angular distribution asymmetry parameters [40].

\section{Discussion}

\subsection{Assessment of the Adiabatic Hyperspherical Approximation}

As we have shown in this chapter, the strengths of the adiabatic hyperspherical description of two-electron processes are several. First, it includes much of the most important electron correlations. Hence adiabatic energies and wave functions are surprisingly accurate for a first-order approximation, particularly for the lowest states in a particular adiabatic potential. Second, the long range dipole interactions between an electron and a hydrogenic atomic or ionic core are diagonalized asymptotically in the adiabatic hyperspherical representation. This permits one to analyze fairly subtle effects of electron correlations in a first-order approximation. Third, by reducing the description of two-electron correlations to an analysis of the various allowed adiabatic channels onc can quite casily discern the most relevant physics. The structure of the energy-independent channel functions $\phi_{\mu}$ gives an impression of the overall physical characleristics of the channels $\mu$, while the potentials $U_{\mu}(R)$ describe very pictorially how the system will respond to photons of different energies.

As we have also shown in this chapter, the adiabatic hyperspherical predictions for photoionization and photodetachmert cross sections are reasonably quantitatively accurate near threshold. At higher photon energies, the cross sections become too low becausc of the excessively strong centrifugal potential barrier in the adiabatic potentials $U_{\mu}(R)$. For multiphoton detachment of $\mathrm{H}^{-}$, we have stressed the importance of semiempirically adjusting the adiabatic potentials so that the ground state wave function has the correct energy [37].

\subsection{Future Prospects}

The conceptual and quantitative advantages of the adiabatic hyperspherical method make it desirable to develop numerical methods which will permit one to improve upon the adiabatic hyperspherical predictions to any desired level of accuracy so that one has a complete theorctical description of two-electron processes. This requires that 
one solves the coupled hyperspherical equations (7). However, calculations of $e-\mathrm{H}^{1} \mathrm{~S}^{e}$ phase shifts $[67,68]$ have shown that improvements upon the adiabatic hyperspherical results are slowly convergent. Another, perhaps not unrelated, numerical difficulty is the slow rate at which the adiabatic potentials and channel functions tend to their (independent electron) asymptotic forms [69-71]. Indeed, Christensen-Dalsgaard [69] demonstrated the dramatic improvement in the $e-\mathrm{H}^{1} \mathrm{~S}^{e}$ phase shift that could be obtained by simply matching the adiabatic hyperspherical wave function onto an independent electron wave function at a finite value of $R$.

While the desirability of combining hyperspherical analyses with R-matrix techniques has been noted [72], it is only recently that interest in this task has been rekindled. Sadeghpour [73] has just presented procedures for combining a coupled adiabatic hyperspherical treatment with multichannel quantum defect theory to obtain $\mathrm{H}^{-}$resonance energies and widths of very high accuracy. Most recently, Tang, Watanabe, and Matsuzawa [74] have outlined an R-matrix scheme for matching hyperspherical close-coupling solutions onto independent particle coordinates. For many encrgies, widths, and phase shifts in doubly and singly excited He they obtain 5-digit agreement with results of other computational methods. Methods such as these will permit theorists not only to make detailed quantitative comparisons with experimental data but also to provide very physical interpretations of two-electron dynamics.

\section{Acknowledgments}

It is my pleasure to acknowledge the hospitality of the Joint Institute for Laboratory Astrophysics and the partial support of a JILA Visiting Fellowship. My group's rescarch reported herein was supported in part by the U.S. Department of Energy, Office of Basic Energy Sciences, Division of Chemical Sciences, under Grant No. DE-FG02-88ER13955.

\section{References}

1. E. C. Kemble, The Fundamental Principles of Quantum Mechanics with Elementary Applications (Dover, New York, 1937) p. 210.

2. P. M. Morse and H. Feshbach, Methods of Theoretical Physics (McGraw-Hill, New York, 1953) Vol. II, pp. 1730-1734.

3. V. A. Fock, lzv. $\Lambda$ kad. Nauk USSR, Ser Fiz. 18 (1954) 161 [Eng. Transl.: Kong. Norske Videnskabers Selskabs Forh. 31 (1958) 183; 31 (1958) 145].

4. Yu.N. Demkov and A. M. Ermolaev, Zh. Eksp. Teor. Fiz. 36 (1959) 896 [Sov. Phys.-JETP 36 (1959) 633].

5. F. T. Smith, Phys. Rev. 120 (1960) 1058.

6. W. Zickendraht, Annals of Physics 35 (1965) 18. 
7. (a) K. Frankowski and C. L. Pekeris, Phys. Rev. 116 (1966) 46; (b) K. Frankowski, Phys. Rev. 160 (1967) 1; (c) D. E. Freund, B. D. Huxtable, and J. D. Morgan, Phys. Rev. A 29 (1984) 980.

8. J. 11. Macek, Phys. Rev. 160 (1967) 170.

9. J. H. Macek, J. Phys. B 2 (1968) 831.

10. U. Fano, Rep. Prog. Phys. 46 (1983) 97-165.

11. C. D. Lin, Adv. At. Mol. Phys. 22 (1986) 77.

12. C. D. Lin, in Fundamental Processes of Atomic Dynamics, edited by J. S. Briggs, II. Kleinpoppen, and H. O. Lutz (Plenum, New York, 1988), pp. 259-273.

13. A. F. Starace, in Fundamental Processes of Atomic Dynamics, edited by J. S. Briggs, H. Kleinpoppen, and H. O. Lutz (Plenum, New York, 1988), pp. 235258.

14. M. Cavagnero, in Fundamental Processes of Atomic Dynamics, edited by J. S. Briggs, H. Kleinpoppen, and H. O. Lutz (Plenum, New York, 1988), pp. 301-310.

15. U. Fano and C. D. Lin, Atomic Physics \& (Plenum, New York, 1975), pp. 47-70.

16. C. D. Lin, Phys. Rev. A 10 (1974) 1986.

17. C. D. Lin, Phys. Rev. A 25 (1982) 76.

18. C. D. Lin, Phys. Rev. A 27 (1983) 22.

19. C. H. Park and A. F. Starace, Phys. Rev. A 29 (1984) 442.

20. R. P. Madden and K. Codling, Astrophys. J. 141 (1965) 364.

21. J. W. Cooper, U. Fano, and F. Prats, Phys. Rev. Lett. 10 (1963) 518.

22. C. H. Park, A. F. Starace, J. Tan, and C. D. Lin, Phys. Rev. A 33 (1986) 1000.

23. D. L. Miller and A. F. Starace, J. Phys. B 13 (1980) L525.

24. J. A. R. Samson, Phys. Reports 28C (1976) 303.

25. V. L. Jacobs, Phys. Rev. A 3 (1971) 289.

26. K. A. Bcrrington, P. G. Burke, W. C. Fon, and K. T. Taylor, J. Phys. B 15 (1982) L603.

27. J. B. West and G. V. Marr, Proc. Roy. Soc. A 349 (1976) 397.

28. C. H. Greene, Phys. Rev. A 23 (1981) 661.

29. J. Dubau and J. Wells, J. Phys. B 6 (1973) 1452. 
30. P. F. O'Mahony and C. H. Greene, Phys. Rev. A 31 (1985) 250.

31. (a) C. H. Greene and L. Kim, Phys. Rev. A 36 (1987) 2706; (b) L. Kim and C. H. Greene, Phys. Rev. A 36 (1987) 4272.

32. M. Aymar, E. Luc-Kocnig, and S. Watanabe, J. Phys. B 20 (1987) 4325.

33. M. G. J. Fink and P. Zoller, J. Phys. B 18 (1985) L373.

34. K. L. Bell and A. E. Kingston, Proc. Phys. Soc. A 90 (1967) 895.

35. M. P. Ajmera and K. T. Chung, Phys. Rev. A 12 (1976) 475.

36. M. Daskhan and A. S. Ghosh, Phys. Rev. A 28 (1983) 2767.

37. C. R. Liu, B. Gao, and A. F. Starace, Phys. Rev. A (to be published).

38. C. H. Park, A. F. Starace, and J. Macek, Phys. Rev. A 31 (1985) 1336.

39. C. L. Pekeris, Phys. Rev. 126 (1962) 1470.

40. C. R. Liu, N. Y. Du, and A. F. Starace, Phys. Rev. A 43 (1991) 5891.

41. K. B. Butterfield, Ph.D Thesis, The University of New Mexico (1984), Sec. IV. Tabulated data for the $n=2$ cross section in Fig. 23 appears on pp. 124 and 125.

42. C. D. Lin, Phys. Rev. Lett. 35 (1975) 1150.

43. J. Macek and P. G. Burke, Proc. Phys. Soc. 92 (1967) 351; J. Macek, ibid, 92 (1967) 365.

44. H. A. Hyman, V. L. Jacobs, and P. G. Burke, J. Phys. B. 5 (1972) 2282.

45. J. T. Broad and W. P. Reinhardt, Phys. Rev. A 14 (1976) 2159 .

46. A. W. Wishart, J. Phys. B 12 (1979) 3511.

47. H. C. Bryant, D. A. Clark, K. B. Butterfield, C. A. Frost, H. Sharifian, H. Tootoonchi, J. B. Donahue, P. A. M. Gram, M. E. Hamm, R. W. Hamm, J. C. Pratt, M. A. Yatcs, and W. W. Smith, Phys. Rev. A 27 (1983) 2889, Sec. VII.

48. H. R. Sadeghpour and C. H. Greene, Phys. Rev. Lett. 65 (1990) 313.

49. P. G. Harris, H. C. Bryant, A. H. Mohagheghi, R. A. Reeder, H. Sharifian, C. Y. Tang, II. Tooloonchi, J. B. Donahue, C. R. Quick, D. C. Rislove, W. W. Smith, and J. E. Stewart, Phys. Rev. Lett. 65 (1990) 309.

50. X. H. Liu, Z. Chen, and C. D. Lin, Phys. Rev. A 44 (1991) 5468.

51. J. M. Rost, J. S. Briggs, and J. M. Feagin, Phys. Rev. Lett. 66 (1991) 1642.

52. J. M. Feagin and J. S. Briggs, Phys. Rev. A 37 (1988) 4599; J. M. Feagin, in Fundamental Proccsses of Atomic Dynamics, edited by J. S. Briggs, H. Kleinpoppen, and H. O. Lutz (Plenum, New York, 1988), pp. 275-300. 
53. J. M. Rost and J. S. Briggs, J. Phys. B 23 (1990) L339.

54. H. R. Sadeghpour, C. H. Greene, and M. Cavagnero, Phys. Rev. A 45 (1992) 1587.

55. M. Domke, C. Xue, A. Puschmann, T. Mandel, E. Hudson, D. A. Shirley, G. Kaindl, C. H. Greene, H. R. Sadeghpour, and H. Petersen, Phys. Rev. Lett. 66 (1991) 1306.

56. H. R. Sadeghpour, Phys. Rev. A 43 (1991) 5821.

57. J. Botero and C. H. Greene, Phys. Rev. Lett. 56 (1986) 1366.

58. A. Dalgarno and J. T. Lewis, Proc. R. Soc. London, Ser. A 233 (1955) 70.

59. B. Gao and A. F. Starace, Phys. Rev. Lett. 61 (1988) 404; Phys. Rev. A 39 (1989) 4550.

60. B. Gao, C. Pan, C. R. Liu, and A. F. Starace, J. Opt. Soc. Am. B 7 (1990) 622.

61. S. Geltınan, Phys. Rev. A 43 (1991) 4930.

62. M. Gailitis and R. Damburg, Zh. Eksp. Teor. Fiz. 44 (1963) 1644 (Sov. Phys. JETP 17 (1963) 1107], and Proc. Phys. Soc. London 82 (1963) 192; M. Gailitis, in Atomic Physics 6, edited by R. Damburg and O. Kukaine (Plenum, New York, 1978), pp. 249-266.

63. M. J. Seaton, Proc. Phys. Soc. (London) 77 (1961) 174.

64. C. Greene, U. Fano, and G. Strinati, Phys. Rev. A 19 (1979) 1485.

65. C. D. Lin, Phys. Rev. A 29 (1984) 1019; Adv. At. Mol. Phys. 22 (1986) 77. Specifically, our channels $\mu$ converging to the $\mathrm{H}(\mathrm{n}=2)$ level have the following labels in Lin's $(\mathrm{K}, \mathrm{T})^{A}$ notation: ${ }^{1} \mathrm{~S}(\mathrm{~K}= \pm 1):( \pm 1,0)^{+} ;{ }^{1} \mathrm{P}+:(0,1)^{+} ;{ }^{1} \mathrm{P}-:(1,0)^{-}$; ${ }^{1} \mathrm{P}(\mathrm{pd}):(-1,0)^{0} ;{ }^{1} \mathrm{D}+:(1,0)^{+}$.

66. U. Fano and A. R. P. Rau, Atomic Collisions and Spectra (Academic, New York, 1986), Secs. 5.4 and 5.7.3.

67. C. D. Lin, Phys. Rev. A 12 (1975) 493.

68. L. Pelamourges, S. Watanabe, and M. LeDourneuf, in Electronic and Atomic Collisions: Abstracts of Contributed Papers, XIII ICPEAC, edited by J. Eichler, W. Fritsch, I. V. Hertel, N. Stolterfoht, and U. Wille (Berlin, 1983), p. 109.

69. B. L. Christensen-Dalsgaard, Phys. Rev. A 29 (1984) 2242.

70. Z. Zhen and J. Macek, Phys. Rev. A 34 (1986) 838.

71. D. Cordes and P. L. Altick, Phys. Rev. A 38 (1988) 5479.

72. P. F. O'Mahony and S. Watanabe, J. Phys. B. 18 (1985) L239.

73. II. R. Sadeghpour, J. Phys. B 25 (1992) L29.

74. J. Z. Tang, S. Watanabe, and M. Matsuzawa, Phys. Rev. A (in press). 